\title{
A HIERARCHY OF APPROXIMATIONS OF THE MASTER EQUATION SCALED BY A SIZE PARAMETER
}

\author{
LARS FERM $^{1}$, PER LÖTSTEDT ${ }^{1}$, ANDREAS HELLANDER $^{1}{ }^{*}$ \\ ${ }^{1}$ Division of Scientific Computing, Department of Information Technology \\ Uppsala University, SE-75105 Uppsala, Sweden \\ emails: ferm@it.uu.se, perl@it.uu.se, andreas.hellander@it.uu.se
}

\begin{abstract}
Solutions of the master equation are approximated using a hierarchy of models based on the solution of ordinary differential equations: the macroscopic equations, the linear noise approximation and the moment equations. The advantage with the approximations is that the computational work with deterministic algorithms grows as a polynomial in the number of species instead of an exponential growth with conventional methods for the master equation. The relation between the approximations is investigated theoretically and in numerical examples. The solutions converge to the macroscopic equations when a parameter measuring the size of the system grows. A computational criterion is suggested for estimating the accuracy of the approximations. The numerical examples are models for the migration of people, in population dynamics and in molecular biology.
\end{abstract}

Keywords: master equation, reaction rate equations, linear noise approximation, moment equations

AMS subject classification: 37M05, 34E10, 65C50

\section{Introduction}

The master equation (ME) is an equation for the time evolution of the probability density function (PDF) for the copy numbers of different species in systems with an intrinsic noise $[18,25]$. The systems are modeled as Markov processes with

*Financial support has been obtained from the Swedish Foundation for Strategic Research and the Swedish Graduate School in Mathematics and Computing. 
discrete states in continuous time. Analytical solutions of the ME are known only under simplifying assumptions. Most systems call for a numerical solution of the governing equation. Examples where the ME is used as a model are in stochastic processes in physics and chemistry [17, 20, 25], population dynamics in ecology $[5,11,31]$, the spread of infectuous disease in epidemiology [4, 37], molecular biology [9, 10, 29, 30], and the social sciences [41, 42]. Modeling on a mesoscale level with the ME is necessary in these applications if the copy number of each species is low and stochastic fluctuations are important. If this number is large then a macroscopic equation for the mean values is often satisfactory.

A computational difficulty with the ME is that the dimension of the problem is the same as the number of participating species. The number of different states that the system can reach grows exponentially with increasing number of species making direct numerical solution of the ME impossible for more than a few species. The most popular method for computing the solution is due to Gillespie [20]. His Stochastic Simulation Algorithm (SSA) is a Monte Carlo method for simulation of a time dependent trajectory of a system. From a collection of trajectories, statistical properties can be determined such as mean values, variances, and PDFs. Since SSA is a Monte Carlo method, the convergence is slow in particular if we are interested in the PDF. The progress in time is also slow if the problem has at least two different time scales and the slow scale models the long term dynamics but the fast scale restricts the time steps. Modifications of SSA suitable for stiff problems have been developed [3, 7].

Deterministic algorithms have the advantage of fast convergence and for lowdimensional problems they are superior to SSA [36]. For systems with many species, approximations are necessary for solution of the ME using the moments [12] or the related Fokker-Planck equation [18, 25, 35] to avoid the exponential growth [28]. Usually, there is a size parameter associated with the ME model. It is a measure of the copy numbers of the species or a volume or an area in the model. If the system size is large, the model behaves like a macroscopic system governed by a system of nonlinear ordinary differential equations (ODEs) for the concentrations of the species $[18,25]$. The number of equations is then equal to the number of species and numerical solution is possible for systems with many species. If the copy numbers are small, then only a mesoscopic model given by the ME is sufficiently accurate. In the intermediate range between large and small, there are other equations approximating the ME without suffering from the exponential increase in the computational work. Three of these equations are investigated in this paper and their suitability for numerical approximation of the ME is evaluated.

It is difficult to know a priori if an approximation is accurate or not. The error in the approximation is here quantified by inserting the approximations into the ME. The norm of the residual is a measure of the quality of the solution. Such a posteriori error estimation is found in other applications, see e.g. [33]. The sum in the norm of the residual for high-dimensional problems is computed 
by a Quasi Monte Carlo method [2].

Similar techniques with expansions in a small parameter and moment equations are utilized in high frequency electromagnetics [13]. The moment equations obtained after expansion of the Boltzmann equation in the small Knudsen number are the Euler and Navier-Stokes equations of fluid flow [39]. In turbulence models in computational fluid dynamics [43], higher moments of the fluctuating flow are approximated for closure of the equations. A review article where macroscopic properties are derived from microscopic and stochastic descriptions is found in [21].

In the next section, the $\mathrm{ME}$ and the approximating equations and the relation between them are discussed. The convergence of the solution of the equations for the expected values and the covariances to the solution of the macroscopic equations is proved in Section 3. A method to compute the errors in the ME caused by the approximations is suggested in Section 4. The numerical results are reported in Section 5. The methods are applied to one problem from the social sciences [41], a well known model in population dynamics with foxes and rabbits [32], a system of two metabolites interacting with two enzymes in biochemistry, and a molecular clock in biology $[1,40]$. Conclusions are drawn in the final section.

The notation in the paper is as follows. The $i$ :th element of a vector $\mathbf{v}$ is denoted by $v_{i}$. If $v_{i} \geq 0$ for all $i$, then we write $\mathbf{v} \geq 0$. The Einstein summation convention is adopted so that summation is implied over an index appearing twice in a term. The space derivative of $v_{i}(\mathbf{x})$ with respect to $x_{j}$ is denoted by $v_{i, j}$. The time derivative $\partial p / \partial t$ of $p(\mathbf{x}, t)$ is written $p_{t}$ and $\dot{q}$ is a shorter notation for $d q / d t$. The $\ell_{\alpha}$-norm of $\mathbf{v}$ of length $N$ is $\|\mathbf{v}\|_{\alpha}=\left(\sum_{i=1}^{N}\left|v_{i}\right|^{\alpha}\right)^{1 / \alpha}$. The Euclidean vector norm, $\alpha=2$, is denoted by $\|\mathbf{v}\|=\sqrt{v_{i} v_{i}}$. The corresponding Euclidean norms for multi-index tensors are e.g. $\|\mathbf{A}\|^{2}=A_{i j} A_{i j}=\operatorname{tr} \mathbf{A}^{T} \mathbf{A}$ and $\|\mathbf{B}\|^{2}=B_{i j k} B_{i j k}$. The set of integer numbers is written $\mathbb{Z}$ and $\mathbb{Z}_{+}$denotes the non-negative integer numbers. In the same manner, $\mathbb{R}$ denotes the real numbers and $\mathbb{R}_{+}$is the nonnegative real numbers.

\section{The approximating equations}

The system we are considering has $N$ interacting species $X_{i}, i=1, \ldots, N$, and the copy number of species $X_{i}$ is denoted by $x_{i}$. The state vector $\mathbf{x}$ is timedependent and a transition $r$ from one state $\mathbf{x}_{r}$ to $\mathbf{x}$ occurs with the probability $w_{r}\left(\mathbf{x}_{r}, t\right)$ per time unit. The change in the state vector by a transition is written

$$
\mathbf{x}_{r} \stackrel{w_{r}\left(\mathbf{x}_{r}, t\right)}{\longrightarrow} \mathbf{x}, \quad \mathbf{n}_{r}=\mathbf{x}_{r}-\mathbf{x} .
$$

The difference $\mathbf{n}_{r}$ between the states before and after the transition has only a few non-zero components and $\mathbf{n}_{r} \in \mathbb{Z}^{N}$. In chemistry, $x_{i}$ is the number of molecules of 
species $i$ and $w_{r}\left(\mathbf{x}_{r}, t\right)$ is the propensity for reaction $r$ to take place. In population dynamics, $x_{i}$ is the number of individuals of species $i$. As an example, a new copy of a species may appear or disappear in a transition from one state to another.

The systems are assumed to have a scaling with a size parameter $\Omega$ as in $[25,26]$ which is large compared to 1 and $\varepsilon=\Omega^{-1} \ll 1$. In chemical applications, $\Omega$ can denote a volume and in population dynamics an area. Then $y_{i}=x_{i} / \Omega$ is interpreted as the concentration or population density of species $i$. The propensities for $r=1, \ldots, R$, fulfill the following assumption.

Assumption 1. The propensities have continuous third derivatives in space and time, $w_{r} \in C^{3}[\mathbf{x} \geq 0, t \geq 0]$, and can be written

$$
w_{r}(\mathbf{x}, t)=\Omega v_{r}\left(\Omega^{-1} \mathbf{x}, t\right)=\Omega v_{r}(\mathbf{y}, t),
$$

where $v_{r}$ and its derivatives in $y_{i}$ are of $\mathcal{O}(1)$.

Equations for simulation of the dynamics of a system with transitions between states (1) occurring with probabilities $w_{r}$ satisfying Assumption 1 are given below.

\subsection{The master equation}

At a mesoscopic level with intrinsic noise in the system, the probability density function $(\mathrm{PDF}) p(\mathbf{x}, t)$ for the system to be in the state $\mathbf{x}$ at time $t$ satisfies the $\mathrm{ME}[18,25]$ and $x_{i}$ is a non-negative integer number, $\mathbf{x} \in \mathbb{Z}_{+}^{N}$. Introduce a splitting of $\mathbf{n}_{r}$ into two parts so that

$$
\mathbf{n}_{r}=\mathbf{n}_{r}^{+}+\mathbf{n}_{r}^{-}, n_{r i}^{+}=\max \left(n_{r i}, 0\right), n_{r i}^{-}=\min \left(n_{r i}, 0\right) .
$$

Then the ME for $R$ different transitions is

$$
\begin{aligned}
p_{t}(\mathbf{x}, t) & =\sum_{\substack{r=1 \\
\mathbf{x}+\mathbf{n}_{r}^{-} \geq 0}}^{R} w_{r}\left(\mathbf{x}+\mathbf{n}_{r}, t\right) p\left(\mathbf{x}+\mathbf{n}_{r}, t\right)-\sum_{\substack{r=1 \\
\mathbf{x}-\mathbf{n}_{r}^{+} \geq 0}}^{R} w_{r}(\mathbf{x}, t) p(\mathbf{x}, t) \\
& \equiv(\mathcal{M} p)(\mathbf{x}, t),
\end{aligned}
$$

defining the master operator $\mathcal{M}$. The total probability $\sum_{\mathbf{x} \in \mathbb{Z}_{+}^{N}} p(\mathbf{x}, t)$ is preserved in time with these boundary conditions at $x_{i}=0$ [16].

Direct numerical solution of the ME after approximation of the time derivative is prohibitive if $N$ is greater than four or five. Suppose that the maximum copy number for all species is $x_{\max }$. Then the size of the state space is $\left(x_{\max }+1\right)^{N}$ and it grows exponentially with $N$. Already with $x_{\max }=100$ and $N=5$ the state space is quite large.

\subsection{The macroscopic equation}

The equations for the expected values $m_{i}$ of $x_{i}$ with the PDF satisfying (2) are a system of nonlinear ODEs. They are the governing equations of the dynamics of the system at a macroscopic level. 
By multiplying (2) by $x_{i}$ and summing over $\mathbb{Z}_{+}^{N}$, the equation for $m_{i}=E\left[X_{i}\right] \in$ $\mathbb{R}$ is $[12,25]$

$$
\dot{m}_{i}=-n_{r i} E\left[w_{r}(\mathbf{X}, t)\right]
$$

If every $w_{r}$ is linear in $\mathbf{X}$ then

$$
E\left[w_{r}(\mathbf{X}, t)\right]=w_{r}(E[\mathbf{X}], t)=w_{r}(\mathbf{m}, t),
$$

and the system (3) is closed. If this is not the case, then the macroscopic approximation is

$$
E\left[w_{r}(\mathbf{X}, t)\right] \approx w_{r}(\mathbf{m}, t)
$$

and we arrive at

$$
\dot{m}_{i}=-n_{r i} w_{r}(\mathbf{m}, t) \equiv \omega_{i}(\mathbf{m}, t) .
$$

These are the reaction rate equations in chemical kinetics.

The corresponding equations for the mean values $\mu_{i}=m_{i} / \Omega$ of the concentrations $y_{i}$ follow from Assumption 1

$$
\dot{\mu}_{i}=-n_{r i} v_{r}(\boldsymbol{\mu}, t) \equiv \nu_{i}(\boldsymbol{\mu}, t) .
$$

The number of equations is $N$ and the computational work to solve (4) or (5) grows as a polynomial in $N$ of low degree depending on $\boldsymbol{\omega}$ or $\boldsymbol{\nu}$ and the numerical method.

\subsection{The linear noise approximation}

van Kampen simplified the ME by a Taylor expansion of the state variables $x_{i}$ around the mean values $\mu_{i}$ of the concentrations and $\xi_{i}$ modeling the stochastic variation about $\mu_{i}$ as in $[9,24,25]$. The fluctuations in $\mathbf{x}$ are of $\mathcal{O}\left(\Omega^{1 / 2}\right)$ and the relation between $\mathbf{x}, \boldsymbol{\mu}$, and $\boldsymbol{\xi}$ is

$$
\mathbf{x}=\Omega \mathbf{y}=\Omega\left(\boldsymbol{\mu}+\Omega^{-1 / 2} \boldsymbol{\xi}\right)
$$

with $\mathbf{y}, \boldsymbol{\mu}, \boldsymbol{\xi} \in \mathbb{R}^{N}$. Replace $\mathbf{x}$ in the ME and use Assumption 1 to obtain

$$
p_{t}(\Omega \mathbf{y}, t)=\sum_{r} \Omega\left(v_{r}\left(\mathbf{y}+\Omega^{-1} \mathbf{n}_{r}, t\right) p\left(\Omega\left(\mathbf{y}+\Omega^{-1} \mathbf{n}_{r}\right), t\right)-v_{r}(\mathbf{y}, t) p(\Omega \mathbf{y}, t)\right) .
$$

Introduce

$$
\Pi(\boldsymbol{\xi}, t)=p\left(\Omega \boldsymbol{\mu}+\Omega^{1 / 2} \boldsymbol{\xi}, t\right)=p(\mathbf{x}, t)
$$


into (7) and expand the right hand side into a Taylor series around $\boldsymbol{\mu}$. Let the sum of terms proportional to $\Omega^{1 / 2}$ be zero. Then we arrive at the macroscopic equation (5) for $\boldsymbol{\mu}$.

The terms of $\mathcal{O}(1)$ in (7), ignoring terms of order $\Omega^{-1 / 2}$ and smaller, are

$$
\Pi_{t}=\sum_{r} \Pi v_{r, i} n_{r i}+\Pi_{, i} n_{r i} v_{r, j} \xi_{j}+0.5 v_{r} n_{r i} n_{r j} \Pi_{, i j},
$$

where $v_{r}$ and $v_{r, i}$ are evaluated at $\boldsymbol{\mu}(t)$ from (5). With

$$
V_{i j}(\boldsymbol{\mu}, t)=\sum_{r} n_{r i} n_{r j} v_{r}(\boldsymbol{\mu}, t)
$$

the PDE for $\Pi(\boldsymbol{\xi}, t)$ is simplified to

$$
\Pi_{t}=-\nu_{i, j}\left(\xi_{j} \Pi\right)_{, i}+0.5 V_{i j} \Pi_{, i j} .
$$

It is shown in [24], [35, p. 156] that the solution $\Pi$ of (9) is the PDF of a normal distribution

$$
\begin{aligned}
\Pi(\boldsymbol{\xi}, t) & =\frac{1}{(2 \pi)^{N / 2} \sqrt{\operatorname{det} \Sigma}} \exp \left(-0.5 \xi_{i}\left(\boldsymbol{\Sigma}^{-1}\right)_{i j} \xi_{j}\right) \\
& =\frac{1}{(2 \pi)^{N / 2} \sqrt{\operatorname{det} \boldsymbol{\Sigma}}} \exp \left(-0.5\left(x_{i}-\Omega \mu_{i}\right)\left((\Omega \boldsymbol{\Sigma})^{-1}\right)_{i j}\left(x_{j}-\Omega \mu_{j}\right)\right),
\end{aligned}
$$

where the covariance matrix is the solution of

$$
\dot{\Sigma}_{i j}=\nu_{i, k} \Sigma_{k j}+\nu_{j, k} \Sigma_{k i}+V_{i j} .
$$

The definitions of $\nu_{i, j}(\boldsymbol{\mu}(t), t)$ and $V_{i j}(\boldsymbol{\mu}(t), t)$ are found in (5) and (8). The linear noise approximation (LNA) consists of a normal distribution with variance $\boldsymbol{\Sigma}$ in (11) following the track of the expected value $\Omega \boldsymbol{\mu}$ satisfying the macroscopic equation (5). The dynamics of LNA in (5) and (11) is insensitive to $\Omega$.

There are $N$ non-linear ODEs to solve in (5) for $\boldsymbol{\mu}$. The covariance matrix $\boldsymbol{\Sigma}$ is symmetric and we have to solve $(N+1) N / 2$ linear ODEs in (11). The alternative to solve the PDE in (9) with a standard deterministic algorithm suffers from the exponential growth in $N$ in work and storage.

\subsection{The two-moment equations}

Let us return to (3) for a more accurate approximation of the right hand side than in (4) and let $w_{r}$ satisfy Assumption 1. Then $\omega_{i}(\mathbf{x}, t)$ in (4) shares this property and expand this function about the mean value $\mathbf{m}$ as in [12]

$$
\begin{aligned}
\omega_{i}(\mathbf{x}, t)= & \omega_{i}(\mathbf{m}, t)+\omega_{i, j}\left(x_{j}-m_{j}\right)+0.5 \omega_{i, j k}\left(x_{j}-m_{j}\right)\left(x_{k}-m_{k}\right) \\
& +\rho_{i j k l}\left(x_{j}-m_{j}\right)\left(x_{k}-m_{k}\right)\left(x_{l}-m_{l}\right), \\
\rho_{i j k l}= & 0.5 \int_{0}^{1}(1-\zeta)^{2} \omega_{i, j k l}(\mathbf{m}+\zeta(\mathbf{x}-\mathbf{m}), t) d \zeta .
\end{aligned}
$$


This form of the remainder term is found in [6, p. 190]. Then the expected value of $\omega_{i}(\mathbf{X}, t)$ is

$$
\begin{aligned}
E\left[\omega_{i}(\mathbf{X}, t)\right] & =\omega_{i}(\mathbf{m}, t)+0.5 \omega_{i, j k} \sum_{\mathbf{x}}\left(x_{j}-m_{j}\right)\left(x_{k}-m_{k}\right) p(\mathbf{x}, t)+\eta_{i}, \\
\eta_{i} & =\sum_{\mathbf{x}} \rho_{i j k l}\left(x_{j}-m_{j}\right)\left(x_{k}-m_{k}\right)\left(x_{l}-m_{l}\right) p(\mathbf{x}, t) .
\end{aligned}
$$

The covariance matrix $\mathbf{C}$ is symmetric and defined by

$$
C_{i j}=E\left[\left(X_{i}-m_{i}\right)\left(X_{j}-m_{j}\right)\right]=\sum_{\mathbf{x}}\left(x_{i}-m_{i}\right)\left(x_{j}-m_{j}\right) p(\mathbf{x}, t) .
$$

Thus, a more accurate equation than (4) for $m_{i}$ is derived from (3) (cf. [12])

$$
\dot{m}_{i}=\omega_{i}(\mathbf{m}, t)+0.5 \omega_{i, j k}(\mathbf{m}, t) C_{j k}+\eta_{i} .
$$

The remainder term $\eta_{i}$ vanishes if $\boldsymbol{\omega}$ is at most quadratic in $\mathbf{x}$.

According to [12], $C_{i j}$ satisfies

$$
\begin{aligned}
\dot{C}_{i j} & =E\left[\left(X_{i}-m_{i}\right) \omega_{j}(\mathbf{X}, t)\right]+E\left[\left(X_{j}-m_{j}\right) \omega_{i}(\mathbf{X}, t)\right]+E\left[W_{i j}(\mathbf{X}, t)\right], \\
W_{i j}(\mathbf{x}, t) & =\sum_{r} n_{r i} n_{r j} w_{r}(\mathbf{x}, t) .
\end{aligned}
$$

With the same expansion as in (12), we have that

$$
\begin{aligned}
E\left[\left(X_{i}-m_{i}\right) \omega_{j}(\mathbf{X}, t)\right] & =\omega_{j, k} C_{i k}+Q_{i j}, \\
Q_{i j} & =\sum_{\mathbf{x}} R_{j k l}\left(x_{i}-m_{i}\right)\left(x_{k}-m_{k}\right)\left(x_{l}-m_{l}\right) p(\mathbf{x}, t), \\
R_{j k l} & =\int_{0}^{1}(1-\zeta) \omega_{j, k l}(\mathbf{m}+\zeta(\mathbf{x}-\mathbf{m}), t) d \zeta,
\end{aligned}
$$

and

$$
\begin{aligned}
E\left[W_{i j}\right] & =W_{i j}(\mathbf{m}, t)+0.5 W_{i j, k l} C_{k l}+S_{i j}, \\
S_{i j} & =\sum_{\mathbf{x}} R_{i j k l \ell}\left(x_{k}-m_{k}\right)\left(x_{l}-m_{l}\right)\left(x_{\ell}-m_{\ell}\right) p(\mathbf{x}, t), \\
R_{i j k l \ell} & =0.5 \int_{0}^{1}(1-\zeta)^{2} W_{i j, k l \ell}(\mathbf{m}+\zeta(\mathbf{x}-\mathbf{m}), t) d \zeta .
\end{aligned}
$$

Hence, (14) can be written

$$
\dot{C}_{i j}=\omega_{j, k} C_{i k}+\omega_{i, k} C_{j k}+W_{i j}(\mathbf{m}, t)+0.5 W_{i j, k l} C_{k l}+Q_{i j}+Q_{j i}+S_{i j} .
$$

If $w_{r}$ is at most quadratic in $\mathbf{x}$ then $S_{i j}=0$ in (15), $\eta_{i}=0$ in (13), and $R_{j k l}$ in $Q_{i j}$ depends only on time. Then $Q_{i j}=R_{j k l} D_{i k l}$, where $D_{i k l}$ is the third central moment. The ODE for $D_{i k l}$ has terms including the fourth moment and so on. For closure we assume that central moments of order higher than three are negligible. Then the approximation of $p$ is also a normal distribution as in (10)

$$
\hat{p}(\mathbf{x}, t)=\frac{1}{(2 \pi)^{N / 2} \sqrt{\operatorname{det} \mathbf{C}}} \exp \left(-0.5\left(x_{i}-m_{i}\right)\left(\mathbf{C}^{-1}\right)_{i j}\left(x_{j}-m_{j}\right)\right),
$$


It follows from Assumption 1 that by replacing $\boldsymbol{\omega}$ and $W$ by $\boldsymbol{\nu}$ and $V$ and neglecting $Q_{i j}$ and $S_{i j}$ in (15) we arrive at

$$
\dot{C}_{i j}=\nu_{j, k} C_{i k}+\nu_{i, k} C_{j k}+\Omega V_{i j}(\mathbf{m} / \Omega, t)+0.5 \Omega^{-1} V_{i j, k l} C_{k l} .
$$

If $C_{i j}(\mathbf{y}, 0)$ is of $\mathcal{O}(\Omega)$, then from (17) we conclude that $C_{i j}(\mathbf{y}, t)$ will remain of $\mathcal{O}(\Omega)$ in a finite time interval. With $\boldsymbol{\Sigma}=\Omega^{-1} \mathbf{C}$, the ODE for $\boldsymbol{\Sigma}$ is

$$
\dot{\Sigma}_{i j}=\nu_{j, k} \Sigma_{i k}+\nu_{i, k} \Sigma_{j k}+V_{i j}+0.5 \varepsilon V_{i j, k l} \Sigma_{k l} .
$$

The difference from the equation in (11) is the last term. Introduce the same scaling of $\mathbf{m}$ and $\mathbf{C}$ in (13). Then $\boldsymbol{\mu}$ satisfies

$$
\dot{\mu}_{i}=\nu_{i}(\boldsymbol{\mu}, t)+0.5 \varepsilon \nu_{i, j k}(\boldsymbol{\mu}, t) \Sigma_{j k},
$$

which is the reaction rate equation (5) with an additional, small term depending on the variance. The equations (19) and (18) are the two-moment approximation (2MA) of the ME. They differ from the LNA equations in the dependence of $\boldsymbol{\mu}$ on the variance and the dynamics depending on $\varepsilon$ and $\Omega$.

\section{Analysis of the approximating equations}

The stochastic variable $\mathbf{X}$ with the PDF solving the master equation is first compared to the macroscopic solution in (5). Then the expected values and the covariances obtained with the linear noise approximation (LNA) and the twomoment approximation (2MA) are compared to each other and to the macroscopic solution.

\subsection{The macroscale and the mesoscale models}

Let $X_{i}(t), i=1, \ldots, n$, be the stochastic variables with the PDF $p(\mathbf{x}, t)$ solving (2). Suppose that the propensities satisfy Assumption 1 with the size parameter $\Omega$. If the initial conditions are such that $\lim _{\Omega \rightarrow \infty} \Omega^{-1} \mathbf{X}(0)=\boldsymbol{\mu}(0)$, then it is proved by Kurtz in [26] that

$$
\lim _{\Omega \rightarrow \infty} P\left(\sup _{s \leq t}\left\|\Omega^{-1} \mathbf{X}(s)-\boldsymbol{\mu}(s)\right\|>\delta\right)=0
$$

for any $\delta>0$ in a finite time interval. For large $\Omega$, the stochastic variables are very well approximated by the mean values from the macroscopic model. The result is similar to the law of large numbers.

In another theorem in [27], Kurtz derives an estimate resembling the central limit theorem. Let $\mathbf{Z}_{\Omega}(t)$ be the scaled difference between $\Omega^{-1} \mathbf{X}(t)$ and $\boldsymbol{\mu}(t)$

$$
\Omega^{-1 / 2} \mathbf{Z}_{\Omega}=\Omega^{-1} \mathbf{X}-\boldsymbol{\mu},
$$

cf. (6). Suppose that the initial conditions on $\mathbf{X}$ and $\boldsymbol{\mu}$ are as above. Then $\mathbf{Z}_{\Omega}$ converges in a finite interval when $\Omega \rightarrow \infty$ to a Gaussian diffusion $\mathbf{Z}(t)$ with mean 0 and a covariance depending on $\mathbf{V}$ (see Section 3.2). 


\subsection{The LNA and the 2MA}

The equation for the expected values and the second moments for the 2MA are in Section 2.4

$$
\begin{aligned}
\dot{\mu}_{i} & =\nu_{i}(\boldsymbol{\mu}, t)+0.5 \varepsilon \nu_{i, j k}(\boldsymbol{\mu}, t) \Sigma_{j k}, \\
\dot{\Sigma}_{i j} & =\nu_{j, k}(\boldsymbol{\mu}, t) \Sigma_{i k}+\nu_{i, k}(\boldsymbol{\mu}, t) \Sigma_{j k}+V_{i j}(\boldsymbol{\mu}, t)+0.5 \varepsilon V_{i j, k l}(\boldsymbol{\mu}, t) \Sigma_{k l} .
\end{aligned}
$$

Proposition 1. Assume that the propensities satisfy Assumption 1. Then a solution $\boldsymbol{\mu}, \boldsymbol{\Sigma}$ exists to (22) for any $\varepsilon$ in an interval around $t=0$.

Proof. By the assumption, $\nu_{i}, \nu_{j, k}, \nu_{i, j k}, V_{i j}$, and $V_{i j, k l}$ are continuously differentiable and the result follows from [6, p. 283].

Let us expand the solution of (22) in the small parameter $\varepsilon$

$$
\begin{aligned}
\mu_{i} & =\mu_{0 i}+\varepsilon \mu_{1 i}, \\
\Sigma_{i j} & =\Sigma_{0 i j}+\varepsilon \Sigma_{1 i j} .
\end{aligned}
$$

The terms $\mu_{0 i}$ and $\Sigma_{0 i j}$ in (23) satisfy the equations given by the terms of zero order in $\varepsilon$ in $(22)$

$$
\begin{aligned}
\dot{\mu}_{0 i} & =\nu_{i}\left(\boldsymbol{\mu}_{0}, t\right), \\
\dot{\Sigma}_{0 i j} & =\nu_{j, k}\left(\boldsymbol{\mu}_{0}, t\right) \Sigma_{0 i k}+\nu_{i, k}\left(\boldsymbol{\mu}_{0}, t\right) \Sigma_{0 j k}+V_{i j}\left(\boldsymbol{\mu}_{0}, t\right) .
\end{aligned}
$$

These are the equations (5) and (11) for the moments in the linear noise approximation in Section 2.3.

Let $\delta_{i j}$ be the Kronecker tensor and let $\Phi_{i j}(t, s), t \geq s$, satisfy

$$
\dot{\Phi}_{i j}(t, s)=\nu_{i, k} \Phi_{k j}, \Phi_{i j}(s)=\delta_{i j},
$$

with the solution

$$
\Phi_{i j}(t, s)=\left(\exp \left(\int_{s}^{t} \boldsymbol{\nu}^{\prime}(u) d u\right)\right)_{i j}
$$

where $\boldsymbol{\nu}^{\prime}$ is the gradient matrix with $\left(\boldsymbol{\nu}^{\prime}\right)_{i j}=\nu_{i, j}$. Using (25), the covariance $\Sigma_{0}$ with the initial condition $\Sigma_{0}(0)$ in $(24 \mathrm{~b})$ with $\Phi_{i j}=\Phi_{i j}(t, 0)$ can be written explicitly [24]

$$
\begin{aligned}
\Sigma_{0 i j}(t) & =\Phi_{i k} \Sigma_{0 k l}(0) \Phi_{l j}^{T}+\Phi_{i k} \int_{0}^{t}\left(\Phi^{-1}\right)_{k l} V_{l \ell}\left(\Phi^{-T}\right)_{\ell m} d s \Phi_{m j}^{T} \\
& =\Phi_{i k} \Sigma_{0 k l}(0) \Phi_{l j}^{T}+\int_{0}^{t} \Phi_{i l}(t, s) V_{l \ell}(s) \Phi_{\ell j}^{T}(t, s) d s .
\end{aligned}
$$

The mean value $\boldsymbol{\mu}$ in (21) satisfies (24a) and the expression for the covariances of $\mathbf{Z}$ is (26) [14, Ch. 11] and it satisfies (24b). 
For the boundedness of $\boldsymbol{\Sigma}_{0}$ when $t$ grows we need a second assumption.

Assumption 2. The propensities are such that

$$
x_{i} \nu_{i, j} x_{j} \leq \kappa x_{i} x_{i}, \kappa<0,
$$

in a neighborhood of $\boldsymbol{\mu}_{0}$ for all $t \geq 0$ and any $\mathbf{x}$.

The stability of $\mu_{0 i}$ and $\Sigma_{0 i j}$ in LNA when $t \rightarrow \infty$ is guaranteed in the following proposition. A quantity is bounded if it is bounded in the $\|\cdot\|$-norm.

Proposition 2. Assume that the propensities satisfy Assumptions 1 and 2 when $t \in \mathcal{T}=[0, \infty)$ and that $\boldsymbol{\mu}_{0}$ in (24a) is bounded in $\mathcal{T}$. Then $\boldsymbol{\Sigma}_{0}$ is also bounded in $\mathcal{T}$. Let $\boldsymbol{\mu}_{0}+\delta \boldsymbol{\mu}$ be the solution of a perturbed (24a)

$$
\dot{\mu}_{0 i}+\dot{\delta \mu_{i}}=\nu_{i}\left(\boldsymbol{\mu}_{0}+\delta \boldsymbol{\mu}, t\right)+\tau_{i}, \delta \boldsymbol{\mu}(0)=\delta \boldsymbol{\mu}_{0} .
$$

If the term $\boldsymbol{\tau}$ on the right hand side is bounded in $\mathcal{T}$ and $\delta \boldsymbol{\mu}_{0}$ is so small that Assumption 2 is valid at $t=0$, then $\delta \boldsymbol{\mu}$ is also bounded in $\mathcal{T}$.

Proof. Multiply (24) by $\Sigma_{0 i j}$ to obtain

$$
\begin{aligned}
\Sigma_{0 i j} \dot{\Sigma}_{0 i j} & =\left\|\boldsymbol{\Sigma}_{0}\right\|\left\|\boldsymbol{\Sigma}_{0}\right\|_{t}=\Sigma_{0 i j} \nu_{j, k} \Sigma_{0 k i}+\Sigma_{0 j i} \nu_{i, k} \Sigma_{0 k j}+\Sigma_{0 i j} V_{i j}\left(\boldsymbol{\mu}_{0}, t\right) \\
& \leq 2 \kappa\left\|\boldsymbol{\Sigma}_{0}\right\|^{2}+\left\|\boldsymbol{\Sigma}_{0}\right\|\|\mathbf{V}\|,
\end{aligned}
$$

using the Cauchy-Schwarz inequality. Thus,

$$
\left\|\boldsymbol{\Sigma}_{0}\right\|_{t} \leq 2 \kappa\left\|\boldsymbol{\Sigma}_{0}\right\|+\|\mathbf{V}\|
$$

and by Gronwall's lemma we have

$$
\left\|\boldsymbol{\Sigma}_{0}\right\|(t) \leq e^{2 \kappa t}\left\|\boldsymbol{\Sigma}_{0}\right\|(0)+\int_{0}^{t} e^{2 \kappa(t-s)}\|\mathbf{V}\|(s) d s \leq e^{2 \kappa t}\left\|\boldsymbol{\Sigma}_{0}\right\|(0)+0.5 \gamma\left(1-e^{2 \kappa t}\right) /|\kappa|,
$$

if $\gamma$ is the bound on $\|\mathbf{V}\|(t)$ in $\mathcal{T}$ and we have the bound on $\boldsymbol{\Sigma}_{0}$.

The perturbation $\delta \boldsymbol{\mu}$ satisfies

$$
\begin{aligned}
\delta \dot{\mu}_{i} & =\nu_{i}\left(\boldsymbol{\mu}_{0}+\delta \boldsymbol{\mu}, t\right)-\nu_{i}\left(\boldsymbol{\mu}_{0}, t\right)+\tau_{i}=\bar{\nu}_{i, j} \delta \mu_{j}+\tau_{i}, \\
\bar{\nu}_{i, j} & =\int_{0}^{1} \nu_{i, j}\left(\boldsymbol{\mu}_{0}+\zeta \delta \boldsymbol{\mu}, t\right) d \zeta .
\end{aligned}
$$

Multiply both sides in (28) by $\delta \mu_{i}$. Since

$$
\delta \mu_{i} \int_{0}^{1} \nu_{i, j} d \zeta \delta \mu_{j} \leq \kappa \delta \mu_{i} \delta \mu_{i}
$$

we can derive an inequality similar to (27) for $\delta \boldsymbol{\mu}$. The solution stays in the neighborhood of $\boldsymbol{\mu}_{0}$ and the claim follows.

Remark. The sufficient condition for bounded perturbations in (24a) is the same as it is for bounded covariances in (24b). If $\boldsymbol{\nu}$ is linear in $\boldsymbol{\mu}$ and independent of 
$t$, then $\nu_{i, j}$ is a constant matrix $A_{i j}$ whose eigenvalues determine the stability of $\delta \boldsymbol{\mu}$ and $\boldsymbol{\Sigma}$. Assumption 2 is then a condition on the sign of the eigenvalues of the symmetric part of $A_{i j}$.

The equations for the first order terms $\mu_{1 i}$ and $\Sigma_{1 i j}$ are derived from (22), (23), and (24)

$$
\begin{aligned}
\dot{\mu}_{1 i}= & \varepsilon^{-1}\left(\nu_{i}\left(\boldsymbol{\mu}_{0}+\varepsilon \boldsymbol{\mu}_{1}, t\right)-\nu_{i}\left(\boldsymbol{\mu}_{0}, t\right)\right)+0.5 \nu_{i, j k}(\boldsymbol{\mu}, t) \Sigma_{j k}, \\
\dot{\Sigma}_{1 i j}= & \varepsilon^{-1}\left(\nu_{j, k}\left(\boldsymbol{\mu}_{0}+\varepsilon \boldsymbol{\mu}_{1}, t\right) \Sigma_{i k}-\nu_{j, k}\left(\boldsymbol{\mu}_{0}, t\right) \Sigma_{0 i k}\right) \\
& +\varepsilon^{-1}\left(\nu_{i, k}\left(\boldsymbol{\mu}_{0}+\varepsilon \boldsymbol{\mu}_{1}, t\right) \Sigma_{j k}-\nu_{i, k}\left(\boldsymbol{\mu}_{0}, t\right) \Sigma_{0 j k}\right) \\
& +\varepsilon^{-1}\left(V_{i j}\left(\boldsymbol{\mu}_{0}+\varepsilon \boldsymbol{\mu}_{1}, t\right)-V_{i j}\left(\boldsymbol{\mu}_{0}, t\right)\right)+0.5 V_{i j, k l}(\boldsymbol{\mu}, t) \Sigma_{k l} .
\end{aligned}
$$

There are three types of terms in (29):

$$
\begin{aligned}
& \left.\nu_{i}\left(\boldsymbol{\mu}_{0}+\varepsilon \boldsymbol{\mu}_{1}, t\right)-\nu_{i}\left(\boldsymbol{\mu}_{0}, t\right)\right)=\varepsilon \int_{0}^{1} \nu_{i, j}\left(\boldsymbol{\mu}_{0}+\zeta \varepsilon \boldsymbol{\mu}_{1}, t\right) d \zeta \mu_{1 j}=\varepsilon \bar{\nu}_{i, j} \mu_{1 j}, \\
& \nu_{j, k}\left(\boldsymbol{\mu}_{0}+\varepsilon \boldsymbol{\mu}_{1}, t\right)\left(\Sigma_{0 i k}+\varepsilon \Sigma_{1 i k}\right)-\nu_{j, k}\left(\boldsymbol{\mu}_{0}, t\right) \Sigma_{0 i k}=\varepsilon \bar{\nu}_{j, k l} \mu_{1 l} \Sigma_{0 i k}+\varepsilon \tilde{\nu}_{j, k} \Sigma_{1 i k}, \\
& \nu_{i, j k}\left(\boldsymbol{\mu}_{0}+\varepsilon \boldsymbol{\mu}_{1}, t\right)\left(\Sigma_{0 j k}+\varepsilon \Sigma_{1 j k}\right)=\tilde{\nu}_{i, j k} \Sigma_{0 j k}+\varepsilon \tilde{\nu}_{i, j k} \Sigma_{1 j k}
\end{aligned}
$$

where a variable $\tilde{\nu}$ is evaluated at $\boldsymbol{\mu}_{0}+\varepsilon \boldsymbol{\mu}_{1}$. With the notation in (30), the equations in (29) for $\boldsymbol{\mu}_{1}$ and $\boldsymbol{\Sigma}_{1}$ are

$$
\begin{aligned}
\dot{\mu}_{1 i}= & \bar{\nu}_{i, j} \mu_{1 j}+0.5 \tilde{\nu}_{i, j k} \Sigma_{0 j k}+0.5 \varepsilon \tilde{\nu}_{i, j k} \Sigma_{1 j k}, \\
\dot{\Sigma}_{1 i j}= & \bar{\nu}_{j, k l} \mu_{1 l} \Sigma_{0 i k}+\tilde{\nu}_{j, k} \Sigma_{1 i k}+\bar{\nu}_{i, k l} \mu_{1 l} \Sigma_{0 j k}+\tilde{\nu}_{i, k} \Sigma_{1 j k} \\
& +\bar{V}_{i j, k} \mu_{1 k}+0.5 \tilde{V}_{i j, k l}\left(\Sigma_{0 k l}+\varepsilon \Sigma_{1 k l}\right) .
\end{aligned}
$$

In a finite time interval, the solution to (31) exists for any $\varepsilon$ :

Proposition 3. Assume that the propensities satisfy Assumption 1. Then a solution $\boldsymbol{\mu}_{1}, \boldsymbol{\Sigma}_{1}$ to (31) exists in an interval around $t=0$ for any $\varepsilon$.

Proof. By the assumption, $\nu_{j, k}, \nu_{i, j k}, V_{i j, k}$, and $V_{i j, k l}$ are continuously differentiable and the claim follows from [6, p. 283].

In a finite interval, $\boldsymbol{\mu}$ and $\boldsymbol{\Sigma}$ will be of $\mathcal{O}(1)$. Thus, the standard deviation of species $i, \sqrt{C_{i i}}$, compared to the mean value $m_{i}$ will behave as

$$
\sqrt{C_{i i}} / m_{i}=\Omega^{1 / 2} \sqrt{\Sigma_{i i}} / \Omega \mu_{i} \sim \Omega^{-1 / 2}
$$

For $\boldsymbol{\mu}_{1}$ and $\boldsymbol{\Sigma}_{1}$ to stay bounded as $t \rightarrow \infty$, a few more assumptions are necessary.

Proposition 4. Assume that the propensities satisfy Assumptions 1 and 2 when $t \in \mathcal{T}=[0, \infty)$ and that $\boldsymbol{\mu}_{0}$ in (24a) is bounded in $\mathcal{T}$. If $\nu_{i, j k}, V_{i j, k}, V_{i j, k l}$, and $\varepsilon$ are sufficiently small, then $\boldsymbol{\mu}_{1}$ and $\boldsymbol{\Sigma}_{1}$ are also bounded in $\mathcal{T}$. 
Proof. By Proposition 2, $\boldsymbol{\Sigma}_{0}$ is bounded. Multiply (31a) by $\mu_{1 i}$ and (31b) by $\Sigma_{1 i j}$, add the equations together, and use Assumption 2 to obtain

$$
\begin{aligned}
& \mu_{1 i} \dot{\mu}_{1 i}+\Sigma_{1 i j} \dot{\Sigma}_{1 i j} \\
& \leq \kappa \mu_{1 i} \mu_{1 i}+2 \kappa \Sigma_{1 i j} \Sigma_{1 i j}+C_{1}\left\|\boldsymbol{\mu}_{1}\right\|\left\|\boldsymbol{\Sigma}_{1}\right\|+C_{2}\left\|\boldsymbol{\mu}_{1}\right\|+\left\|C_{3}\right\| \boldsymbol{\Sigma}_{1}\left\|+\varepsilon C_{4}\right\| \boldsymbol{\Sigma}_{1} \|^{2} \\
& \leq\left(\begin{array}{cc}
\left\|\boldsymbol{\mu}_{1}\right\| \\
\left\|\boldsymbol{\Sigma}_{1}\right\|
\end{array}\right)\left(\begin{array}{cc}
\kappa & C_{1} / 2 \\
C_{1} / 2 & 2 \kappa+\varepsilon C_{4}
\end{array}\right)\left(\begin{array}{l}
\left\|\boldsymbol{\mu}_{1}\right\| \\
\left\|\boldsymbol{\Sigma}_{1}\right\|
\end{array}\right)+\left(C_{2}, C_{3}\right)\left(\begin{array}{c}
\left\|\boldsymbol{\mu}_{1}\right\| \\
\left\|\boldsymbol{\Sigma}_{1}\right\|
\end{array}\right),
\end{aligned}
$$

where $C_{1}, C_{2}, C_{3}$, and $C_{4}$ are

$$
\begin{aligned}
& 2\left\|\overline{\boldsymbol{\nu}}^{\prime \prime}\right\|\left\|\boldsymbol{\Sigma}_{0}\right\|+\left\|\overline{\mathbf{V}}^{\prime}\right\|+0.5 \varepsilon\left\|\tilde{\boldsymbol{\nu}}^{\prime \prime}\right\| \leq C_{1}, \quad 0.5\left\|\tilde{\boldsymbol{\nu}}^{\prime \prime}\right\|\left\|\boldsymbol{\Sigma}_{0}\right\| \leq C_{2}, \\
& 0.5\left\|\tilde{\mathbf{V}}^{\prime \prime}\right\|\left\|\boldsymbol{\Sigma}_{0}\right\| \leq C_{3}, 0.5\left\|\tilde{\mathbf{V}}^{\prime \prime}\right\| \leq C_{4} .
\end{aligned}
$$

The first and second derivative tensors are denoted by ' and "above. If $\kappa(2 \kappa+$ $\left.\varepsilon C_{4}\right)>C_{1}^{2} / 4$, then the quadratic form in (32) is negative for all $\left\|\boldsymbol{\mu}_{1}\right\|$ and $\left\|\boldsymbol{\Sigma}_{1}\right\| \neq$ 0 . With $z^{T}=\left(\left\|\boldsymbol{\mu}_{1}\right\|,\left\|\boldsymbol{\Sigma}_{1}\right\|\right)$ there is a bound on $d\|z\|^{2} / d t$

$$
\|z\|\|\dot{z}\| \leq \alpha\|z\|^{2}+\beta\|z\|,
$$

where $\alpha$ is negative. Then the bound on $\|z\|$ follows from Gronwall's lemma as in the proof of Proposition 2.

The expected values and the covariances for the LNA in Section 2.3 and $\boldsymbol{\mu}$ and the covariances of $\mathbf{Z}$ in (21) in Kurtz' central limit theorem satisfy (24). The equation for the expected values is the macroscopic equation in (5). The equations in (24) are the same as in the 2MA in Section 2.4 with $\varepsilon=0$ in (22). The stability of the solutions of (24a) and the boundedness of $\boldsymbol{\Sigma}_{0}$ for long time integration are ensured by Assumption 2 in Proposition 2. The solutions $\boldsymbol{\mu}_{0}, \boldsymbol{\mu}_{1}, \boldsymbol{\Sigma}_{0}$, and $\boldsymbol{\Sigma}_{1}$ exist in a finite interval for any $\varepsilon$ according to Proposition 3. Hence, as $\varepsilon \rightarrow 0$ the expected values $\boldsymbol{\mu}_{0}+\varepsilon \boldsymbol{\mu}_{1}$ and covariances $\boldsymbol{\Sigma}_{0}+\varepsilon \boldsymbol{\Sigma}_{1}$ of the 2MA converge to the corresponding quantities for the LNA. Additional conditions are necessary for long time boundedness in Proposition 4. The equations (22) and (24) are two alternatives to determine the mean $\boldsymbol{\mu}$ and the approximate covariances of $\mathbf{Z}_{\Omega}$ in (21).

\section{Error estimates}

It is difficult to a priori determine if the LNA and 2MA provide accurate solutions to the ME. By inserting a normally distributed PDF with the expected values and the covariances computed by the LNA and the $2 \mathrm{MA}$ into the ME, we obtain an a posteriori measure of the modeling error.

Solve (5) and (11) or (19) and (18) for $\boldsymbol{\mu}$ and $\boldsymbol{\Sigma}$ with an ODE solver for approximations at discrete time points $t^{k}, k=0,1, \ldots$, with the time step $\Delta t^{k}=$ 
$t^{k}-t^{k-1}$. Then with $\mathbf{m}=\Omega \boldsymbol{\mu}$ and $\mathbf{C}=\Omega \boldsymbol{\Sigma}$ the PDF at $t^{k}$ is

$$
\hat{p}^{k}=\hat{p}\left(\mathbf{x}, t^{k}\right)=\frac{1}{(2 \pi)^{N / 2} \sqrt{\operatorname{det} \mathbf{C}^{k}}} \exp \left(-\frac{1}{2}\left(\mathbf{x}-\mathbf{m}^{k}\right)^{T}\left(\mathbf{C}^{k}\right)^{-1}\left(\mathbf{x}-\mathbf{m}^{k}\right)\right),
$$

where $\mathbf{m}^{k}$ and $\mathbf{C}^{k}$ are the approximations at $t^{k}$ (cf. (16)).

A $p(\mathbf{x}, t)$, sufficiently smooth in $t$, satisfies a discrete approximation of the ME (2) with the second order trapezoidal method [22] such that at $t=t^{k-1}+0.5 \Delta t^{k}$

$$
p_{t}-\mathcal{M} p-r^{k}=\mathcal{O}\left(\left(\Delta t^{k}\right)^{2}\right), r^{k}=\frac{p^{k}-p^{k-1}}{\Delta t^{k}}-\frac{1}{2} \mathcal{M}\left(p^{k}+p^{k-1}\right) .
$$

In particular, a smooth extension $\hat{p}$ of $\hat{p}^{k}$ from $\ldots, t^{k-2}, t^{k-1}, t^{k}$ to $\left[t^{k-1}, t^{k}\right]$ by interpolation in $t$ satisfies (34)

$$
\hat{p}_{t}-\mathcal{M} \hat{p}=\hat{r}^{k}+\mathcal{O}\left(\left(\Delta t^{k}\right)^{2}\right)=\tau .
$$

The smaller $\tau$ is in (35), the better $\hat{p}$ approximates the solution of the ME. The residual $\hat{r}^{k}$ is computed by insertion of $\hat{p}^{k}$ from (33) in the discretized ME. The term proportional to $\left(\Delta t^{k}\right)^{2}$ in (35) is usually much smaller than $\hat{r}^{k}$ and is controlled by the time steps in the ODE solver. An estimate of the time discretization error is obtained if $\hat{r}^{k}$ is compared with the residual computed with $\hat{p}^{k}$ and $\hat{p}^{k-2}$. If the difference is small then the approximation error due to $\hat{p}^{k}$ in (33) is the dominant part in $\tau$. In such a case, $\hat{r}^{k}$ is a reliable measure of the error in the ME due to the approximation in (33).

The $\ell_{1}$-norm of $\hat{r}^{k}$ is defined by

$$
\hat{\rho}^{k}=\left\|\hat{\mathbf{r}}^{k}\right\|_{1}=\sum_{\mathbf{x} \in \mathcal{D}}\left|\hat{r}^{k}(\mathbf{x})\right|
$$

where $\mathcal{D} \subset \mathbb{Z}_{+}^{N}$ is the finite computational domain. It is computed in the next section in the numerical experiments. When $N$ is small, then summation is possible over all points in $\mathcal{D}$. If $N$ is greater than three (say), then the cost of calculating $\hat{\rho}^{k}$ in (36) is overwhelming. Instead, the sum is computed by a Quasi Monte Carlo $(\mathrm{QMC})$ method [2] in a box $\mathcal{D}_{0} \subset \mathcal{D}$ with center at the mean values and extended two standard deviations in both directions along the coordinate axes. Then $\hat{\rho}^{k}$ is approximated by

$$
\hat{\rho}^{k} \approx \frac{\left|\mathcal{D}_{0}\right|}{L} \sum_{l=1}^{L}\left|\hat{r}^{k}\left(\mathbf{x}_{l}\right)\right| .
$$

The volume of $\mathcal{D}_{0}$ is here denoted by $\left|\mathcal{D}_{0}\right|$ and $L$ is the number of quadrature points $\mathbf{x}_{l}$. The points $\mathbf{x}_{l} \in \mathcal{D}_{0}$ are chosen using Faure sequences [15] generated with the algorithm in [23] and $L$ is much smaller than the number of integer points in $\mathcal{D}_{0}$ and $\mathcal{D}$. The error in $\hat{\rho}^{k}$ decays as $\mathcal{O}\left((\log L)^{N} / L\right)[2]$. 
Let $p$ be the analytical solution satisfying $p_{t}-\mathcal{M} p=0$. Then the solution error $e=\hat{p}-p$ solves the error equation

$$
e_{t}-\mathcal{M} e=\tau
$$

derived from (35). It can be solved numerically for an error estimate. The error in linear functionals of the solution such as the moments at a final time is controlled by solving the adjoint equation of the ME [19].

The master equation is solved in the two dimensional examples in the next section by integrating in time with the trapezoidal method [22] on a grid where each cell may contain 1,4 , or 16 integer points from $\mathbb{Z}_{+}^{2}$. The solution variable in a cell represents the average of the PDF in its integer points. The computational domain is partitioned into a number of blocks and each block consists of cells of equal size. The jumps in cell size at the block boundaries is allowed to be at most 2. The cell size in a block is adapted dynamically to the solution for best efficiency and accuracy. The solution is well resolved in many parts of the domain by cells with $4 \times 4$ points. The total probability is conserved by the method. The solution algorithm is similar to the algorithm for the Fokker-Planck equation in [16]. The residual $\hat{r}^{k}$ in (36) is weighted by the area of the cell. A forthcoming paper will have a detailed account of the method. For high dimensional problems with $N \geq 4$, the trajectories of the system are generated by Gillespie's SSA [20].

\section{Numerical results}

The time dependent solutions of the ME (2) are compared to the solutions of the equations for the expected values and the covariances given by the LNA (5), (11) or by the 2MA (19), (18) in this section. Four problems are solved:

- A model of the migration of people from $[41,42]$.

- A model from [32] of a predator-prey interaction in population dynamics.

- A model for the creation of two metabolites controled by two enzymes [8].

- A model of a circadian molecular clock from [1, 40].

The ME is solved by the method described in the end of Section 4. The nonlinear systems of ODEs satisfied by the expected values and the covariances in LNA and 2MA are computed by MATLAB's ode15s. The stationary solutions are obtained by integrating the equations until the time derivatives are small.

\subsection{Migration of people}

Two populations $\mathcal{P}_{1}$ and $\mathcal{P}_{2}$ interact with each other and move between two regions $\mathcal{R}_{1}$ and $\mathcal{R}_{2}$. The system has four states $s_{j}^{i}, i, j=1,2$, representing the 
number of people from $\mathcal{P}_{j}$ in $\mathcal{R}_{i}$. The total number of people in $\mathcal{P}_{j}$ is a constant $\Omega=s_{j}^{1}+s_{j}^{2}$. Then the system has two degrees of freedom

$$
x_{1}=\left(s_{1}^{1}-s_{1}^{2}\right) / 2,-\Omega \leq x_{1} \leq \Omega, x_{2}=\left(s_{2}^{1}-s_{2}^{2}\right) / 2,-\Omega \leq x_{2} \leq \Omega .
$$

There is a propensity of someone in a population $\mathcal{P}_{j}$ to move from one region $\mathcal{R}_{i}$ to the other region $\mathcal{R}_{3-i}$ depending on the number of people in the two populations in the first region. The changes of the state of the system can be written as chemical reactions:

$$
\begin{array}{lllll}
S_{1}^{1} & \stackrel{w_{1}}{\longrightarrow} & S_{1}^{2}, & w_{1}=\gamma\left(\Omega-x_{1}\right) \exp \left(\Omega^{-1}\left(\kappa x_{1}-\sigma x_{2}\right)\right), & \mathbf{n}_{1}^{T}=(-1,0), \\
S_{1}^{2} & \stackrel{w_{2}}{\longrightarrow} & S_{1}^{1}, & w_{2}=\gamma\left(\Omega+x_{1}\right) \exp \left(-\Omega^{-1}\left(\kappa x_{1}-\sigma x_{2}\right)\right), & \mathbf{n}_{2}^{T}=(1,0), \\
S_{2}^{1} & \stackrel{w_{3}}{\longrightarrow} & S_{2}^{2}, & w_{3}=\gamma\left(\Omega-x_{2}\right) \exp \left(\Omega^{-1}\left(-\tau \sigma x_{1}+\kappa x_{2}\right)\right), & \mathbf{n}_{3}^{T}=(0,-1), \\
S_{2}^{2} & \stackrel{w_{4}}{\longrightarrow} & S_{2}^{1}, & w_{4}=\gamma\left(\Omega+x_{2}\right) \exp \left(-\Omega^{-1}\left(-\tau \sigma x_{1}+\kappa x_{2}\right)\right), & \mathbf{n}_{4}^{T}=(0,1),
\end{array}
$$

according to $[41$, p. 87]. In a symmetrical case, $\tau=1$, and in an antisymmetrical case, $\tau=-1$. The propensities satisfy Assumption 1 with

$$
\begin{array}{ll}
v_{1}=\gamma\left(1-y_{1}\right) \exp \left(\kappa y_{1}-\sigma y_{2}\right), & v_{2}=\gamma\left(1+y_{1}\right) \exp \left(-\left(\kappa y_{1}-\sigma y_{2}\right)\right), \\
v_{3}=\gamma\left(1-y_{2}\right) \exp \left(-\tau \sigma y_{1}+\kappa y_{2}\right), & v_{4}=\gamma\left(1+y_{2}\right) \exp \left(-\left(-\tau \sigma x_{1}+\kappa x_{2}\right)\right) .
\end{array}
$$

The examples are the four different scenarios with different $\kappa, \sigma$, and $\tau$ from [41, ch. 4].

\subsubsection{Scenario 1}

With the parameters $\kappa=0.2, \sigma=0.5$, and $\tau=1$, the origin is a stable fixed point of the macroscopic equations [41, p. 89]. The interpretation of this scenario is that there is a homogeneous mixture of both populations in both regions in the steady state, since $s_{1}^{1}=s_{2}^{1}=s_{1}^{2}=s_{2}^{2}$ and $x_{1}=x_{2}=0$ is the most probable time-independent state of the system.

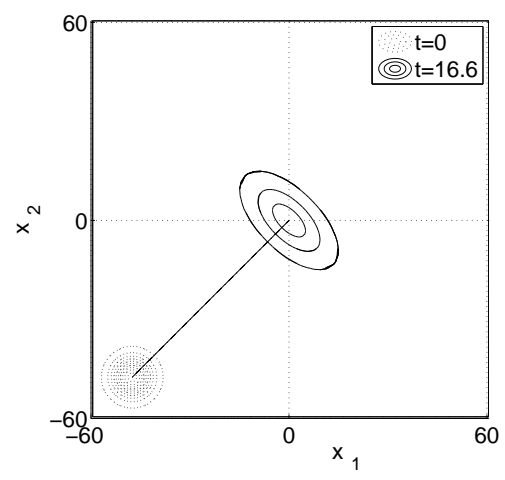

Fig. 1: Scenario 1. Isolines of initial and final ME solutions and coinciding trajectories of the expected values of the ME, LNA, and 2MA. 
The master equation is solved with $\Omega=60$ in Figure 1 starting at $t=0$ with a Gaussian distribution in the lower left corner. An approximate steady state at the origin is reached at $t=16.6$. The trajectories of the expected values from the $\mathrm{ME}, \mathrm{LNA}$, and $2 \mathrm{MA}$ coincide when $t \in[0,16.6]$ in this case and the covariances are

$$
\left(C_{11}, C_{12}, C_{22}\right)= \begin{cases}(59.6,-36.8,59.6) & \mathrm{ME} \\ (61.5,-38.5,61.5) & \text { LNA } \\ (61.2,-38.3,61.2) & 2 \mathrm{MA}\end{cases}
$$

The LNA and 2MA agree very well with the ME solution.

\subsubsection{Scenario 2}

There are two stable fixed points of the macroscopic equation at the upper left and the lower right corners of the $\left(x_{1}, x_{2}\right)$-domain and the origin is unstable if the parameters are $\kappa=0.5, \sigma=1, \tau=1$. The majority of one population prefers one region and the other population prefers the other region.

Starting in the lower left corner with a Gaussian distribution on the diagonal, the ME solution splits into two parts moving into the upper left and lower right corners, see Figure 2. The expected value of the ME, LNA, and 2MA follow the same path to the origin. The covariances of the ME stays bounded but those of LNA and 2MA grow exponentially.

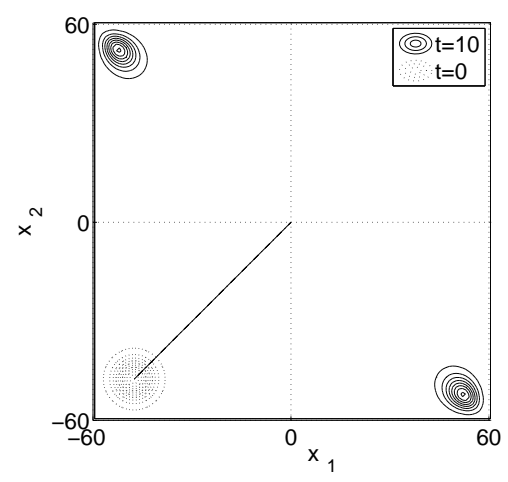

Fig. 2: Scenario 2. Isolines of the ME solution and coinciding trajectories of the expected values of ME, LNA, and 2MA.

In Figure 3, the initial pulse is located asymmetrically in $\left(x_{1}, x_{2}\right)$. The ME solution is distributed between the upper left and the lower right corners. The expected value of the LNA solution reaches the upper left corner and $\Omega \mu$ in $2 \mathrm{MA}$ stops at the origin. The trajectories of the expected values follow the same path to begin with but the steady states of LNA and 2MA differ from the ME result. The ME covariances are $(1370,-1370,1370)$, the LNA covariances $(10,-3,10)$, 
and the 2MA covariances diverge. The fourth central moment is large in this case and is not accounted for by the LNA or 2MA.

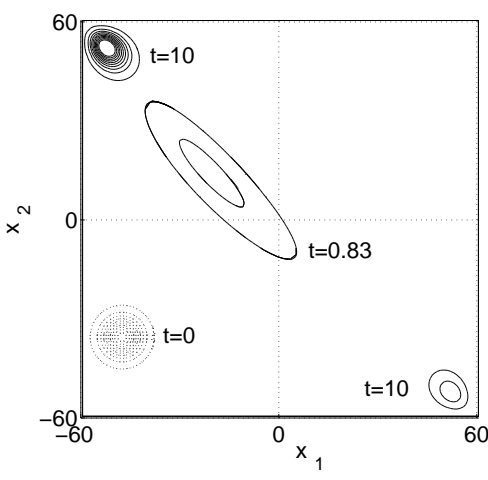

(a)

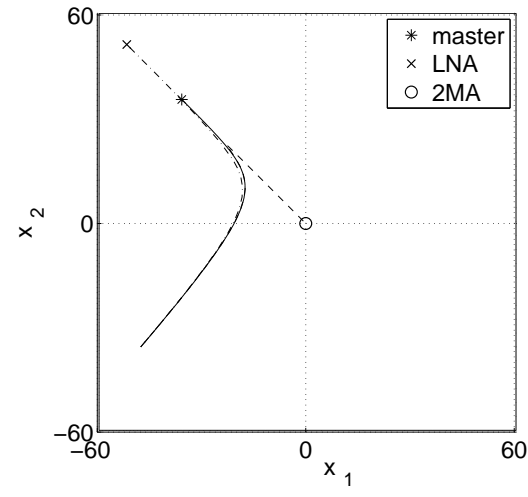

(b)

Fig. 3: Scenario 2. (a) Isolines of the ME solution at different time instants. (b) Trajectories of the expected values of ME, LNA, and 2MA.

\subsubsection{Scenario 3}

There is an asymmetrical interaction between the populations in Scenario 3 with $\kappa=0.5, \sigma=1$, and $\tau=-1$. The origin is a stable fixed point. The population $\mathcal{P}_{1}$ tries to live apart from $\mathcal{P}_{2}$, but $\mathcal{P}_{2}$ wants to live together with $\mathcal{P}_{1}$. The difference from Scenario 1 is that the expected values follow spirals toward the origin.

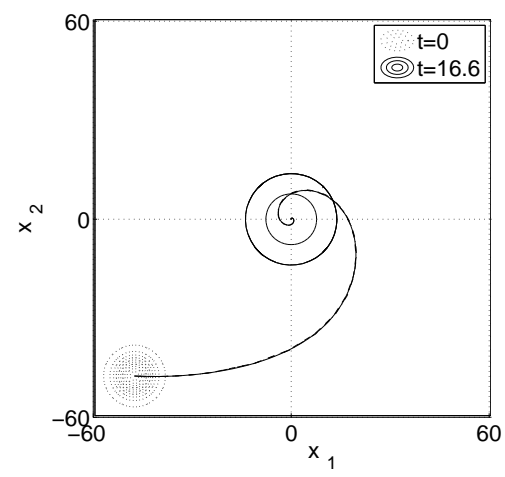

(a)

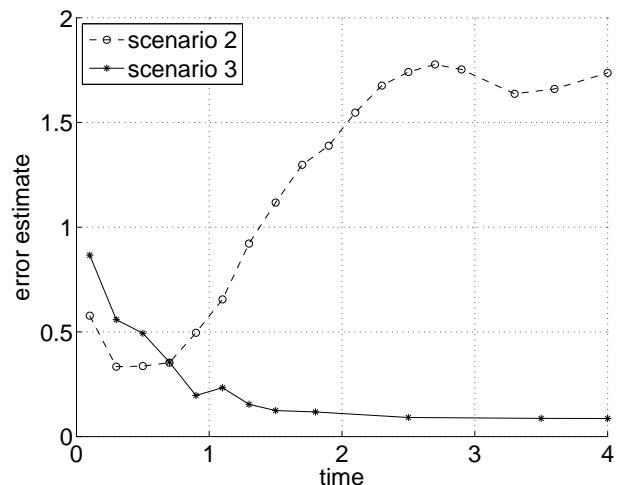

(b)

Fig. 4: Scenario 3. (a) Isolines of the initial and final ME solutions and coinciding trajectories of the expected values of the ME, LNA, and 2MA. (b) Residual error estimates for LNA.

As in Figure 1, there is no visible difference between the trajectories of the 
expected values in Figure 4. The covariances are

$$
\left(C_{11}, C_{12}, C_{22}\right)= \begin{cases}(58.7,0,58.7) & \mathrm{ME} \\ (60.0,0,60.0) & \text { LNA } \\ (60.1,0,60.1) & 2 \mathrm{MA}\end{cases}
$$

The LNA and 2MA agree also here very well with the ME solution. The residual error estimate in Figure 4 is measured as in (36). The figure indicates that LNA a good approximation of ME at least for Scenario 3. When comparing $\rho^{k}$ for two different time steps $\Delta t^{k}$ and $\Delta t^{k}+\Delta t^{k-1}$, the difference is small.

\subsubsection{Scenario 4}

The macroscopic solution approaches a stable limit cycle in the final migration example. If both populations are in the upper right quadrant initially in region $\mathcal{R}_{1}$, then population $\mathcal{P}_{1}$ moves into the upper left quadrant and $\mathcal{R}_{2}$. Population $\mathcal{P}_{2}$ follows to $\mathcal{R}_{2}$ in the lower left quadrant, but then $\mathcal{P}_{1}$ returns to $\mathcal{R}_{1}$. When $\mathcal{P}_{2}$ is back in $\mathcal{R}_{2}$ one period of the solution is complete. The parameters in this case are $\kappa=1.2, \sigma=1$, and $\tau=-1$. The origin is an unstable fixed point.

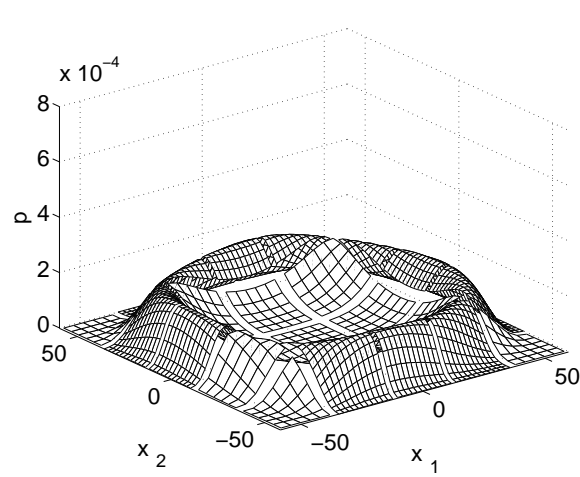

(a)

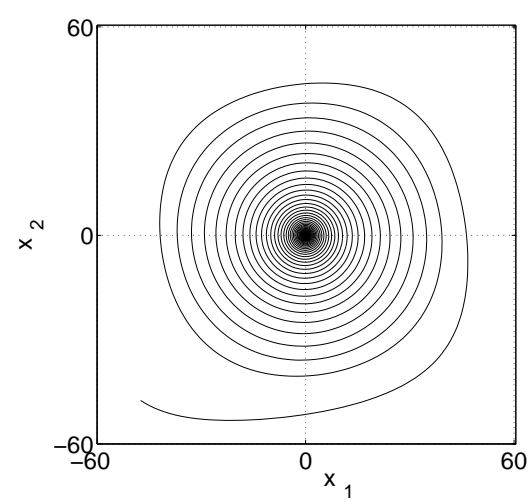

(b)

Fig. 5: The ME solution. (a) The steady state solution and the corresponding adapted grid. (b) The trajectory of the expected value.

The initial state is a Gaussian pulse at the lower left corner in Figure 5. The stationary solution is a crater about the origin. The expected value of the $\mathrm{ME}$ is a spiral ending at the origin. The PDF from the 2MA approximates the ME solution fairly well in Figure 6 in the first period. Then in the second period the covariances start to grow and the PDF from $2 \mathrm{MA}$ is no longer contained in the original domain. 


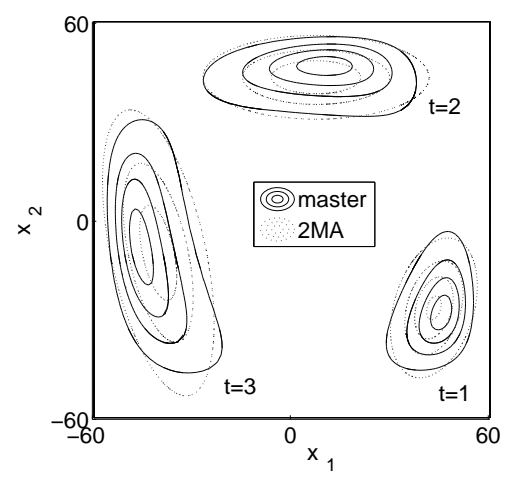

(a)

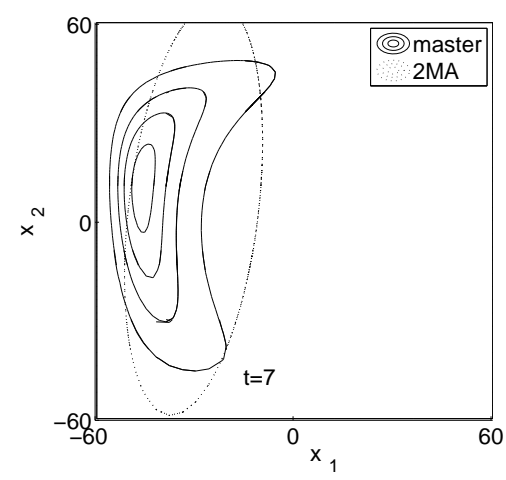

(b)

Fig. 6: Isolines of the ME and 2MA solutions. (a) Three solutions in the first lap around the origin. (b) Solutions at the end of the second lap.

The $\boldsymbol{\mu}_{0}$-value satisfying (5) and (24a) enters a limit cycle in Figure 7 while $\boldsymbol{\mu}$ satisfying (19) and (22) exhibits a spiraling behavior as the ME solution. A breakdown in 2MA occurs before the solution reaches the origin. The 2MA solution is closer to the LNA and the macroscopic solution for a larger $\Omega$ as expected from Proposition 3 in Section 3.

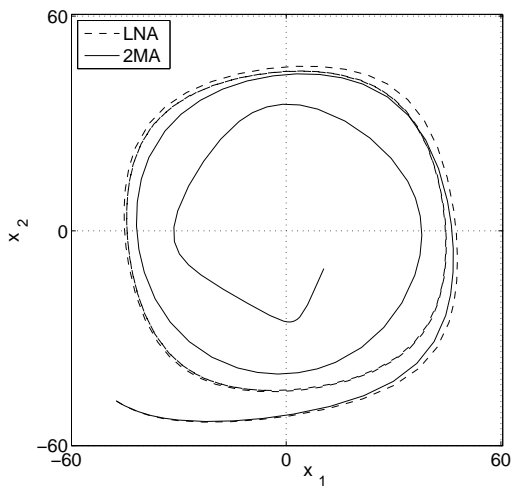

(a)

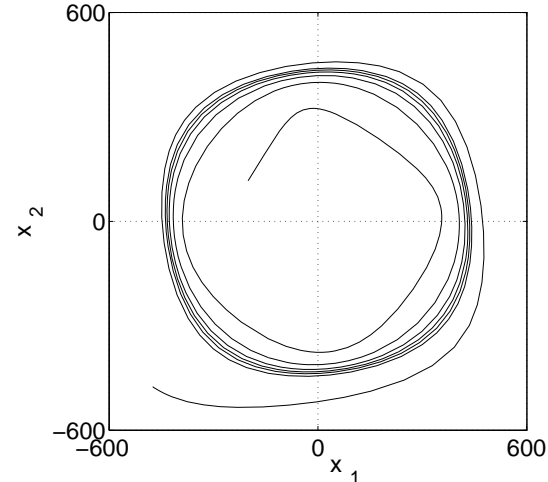

(b)

Fig. 7: Trajectories for the expected values when $\Omega=60$ (a) and $\Omega=600$ (b).

\subsection{The predator-prey problem}

A predator-prey problem in population dynamics is chosen from [32, Ch. 3.4]. Two populations $\mathcal{P}_{1}$ and $\mathcal{P}_{2}$ interact in a limited area. The growth rate of $\mathcal{P}_{1}$ (prey) decreases if the number of $\mathcal{P}_{2}$ (predator) increases and the growth rate of $\mathcal{P}_{2}$ decreases if the number of $\mathcal{P}_{1}$ decreases. An popular example is a forest with rabbits and foxes. 
The propensities for the macroscopic model in [32] satisfying Assumption 1 are

$$
v_{1}=y_{1}, v_{2}=y_{1}^{2}+\frac{a y_{1} y_{2}}{y_{1}+d}, v_{3}=b y_{2}, v_{4}=b \frac{y_{2}^{2}}{y_{1}},
$$

with

$$
\mathbf{n}_{1}^{T}=(-1,0), \mathbf{n}_{2}^{T}=(1,0), \mathbf{n}_{3}^{T}=(0,-1), \mathbf{n}_{4}^{T}=(0,1) .
$$

For some systems it is advantageous to introduce two scaling factors $\Omega_{i}$ if the copy numbers $x_{i}$ differ in magnitude to keep $y_{i}$ of $\mathcal{O}(1)$. The theory in the previous sections covers the case when the scaling of the copy numbers is equal but the solutions in the numerical experiments with $\Omega_{1} / \Omega_{2}=$ const $\neq 1$ converge in the same manner. Let $x_{1}=\Omega_{1} y_{1}$ and $x_{2}=\Omega_{2} y_{2}$. Then the propensities in the ME are written as chemical reactions:

$$
\begin{aligned}
\emptyset \stackrel{w_{1}}{\longrightarrow} X_{1}, \quad w_{1}=\Omega_{1} v_{1}\left(\frac{x_{1}}{\Omega_{1}}, \frac{x_{2}}{\Omega_{2}}\right)=x_{1}, \\
X_{1} \stackrel{w_{2}}{\longrightarrow} \emptyset, \quad w_{2}=\Omega_{1} v_{2}\left(\frac{x_{1}}{\Omega_{1}}, \frac{x_{2}}{\Omega_{2}}\right)=\frac{x_{1}^{2}}{\Omega_{1}}+\frac{\Omega_{1}}{\Omega_{2}} \frac{a x_{1} x_{2}}{x_{1}+\Omega_{1} d}, \\
\emptyset \stackrel{w_{3}}{\longrightarrow} X_{2}, \quad w_{3}=\Omega_{2} v_{3}\left(\frac{x_{1}}{\Omega_{1}}, \frac{x_{2}}{\Omega_{2}}\right)=b x_{2}, \\
X_{2} \stackrel{w_{4}}{\longrightarrow} \emptyset, \quad w_{4}=\Omega_{2} v_{4}\left(\frac{x_{1}}{\Omega_{1}}, \frac{x_{2}}{\Omega_{2}}\right)=b \frac{\Omega_{1}}{\Omega_{2}} \frac{x_{2}^{2}}{x_{1}} .
\end{aligned}
$$

The system is simulated with $\Omega_{2}=0.5 \Omega_{1}$ and two different sets of parameters. The first macroscopic system has a stable fixed point and the second system approaches a stable limit cycle.

\subsubsection{The stable system}

The parameters in the first case are $a=0.75, b=0.2, d=0.2$, in (39). The size parameter $\Omega_{1}$ is 200 in the first example.

The system has two absorbing states: One with extinction of both predator $\left(x_{2}=0\right)$ and prey $\left(x_{1}=0\right)$ and one with no predator $\left(x_{2}=0\right)$ but with some prey $\left(x_{1}>0\right)$. The latter solution can be determined exactly since it is a one dimensional problem in $x_{1}$ at $x_{2}=0$ [18]. The absorbing states are computed as eigensolutions with zero eigenvalues of the master operator $\mathcal{M}$ on a grid with a variable cell size. They have support only on $x_{2}=0$. 


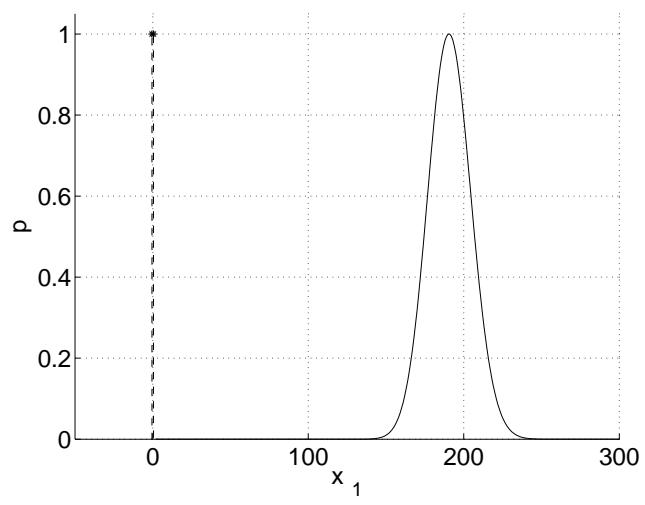

Fig. 8: Two eigensolutions corresponding to the eigenvalue zero.

The solution of the ME is integrated in time from the initial Gaussian distribution centered at $(100,30)$ until it moves very slowly in Figure 9.a. Every sixth grid line is plotted. The solution is close to an eigensolution with an eigenvalue of modulus $10^{-10}$. The trajectory of $2 \mathrm{MA}$ in Figure 9.b is closer to the ME trajectory than the macroscopic and LNA solutions.

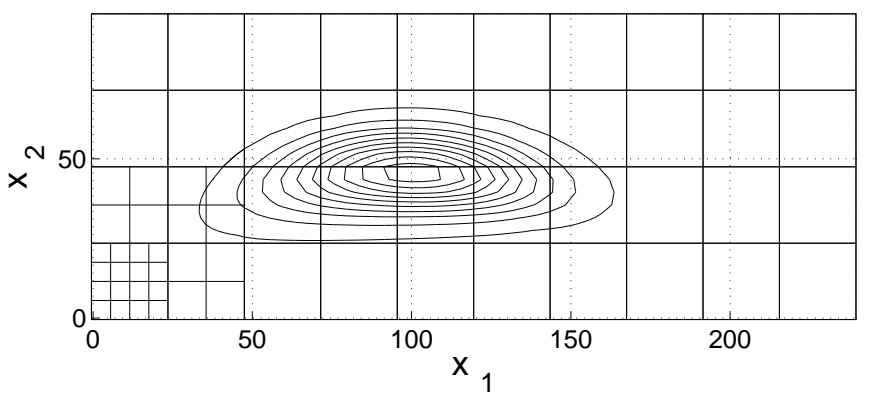

(a)

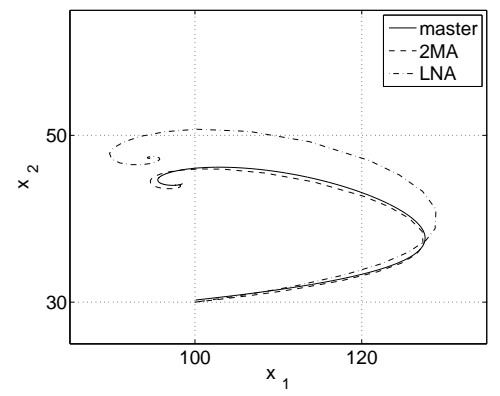

(b)

Fig. 9: The stable problem. (a) Isolines of the ME solution in a quasi-steady state. (b) Trajectories of the expected values.

The expected values follow different paths when $\Omega_{1}=20$ in Figure 10 . The initial solution has its center at $(5,5)$. The LNA solution reaches a steady state in the interior of the domain but both the $\mathrm{ME}$ and the $2 \mathrm{MA}$ find an absorbing state at $x_{2}=0$. 


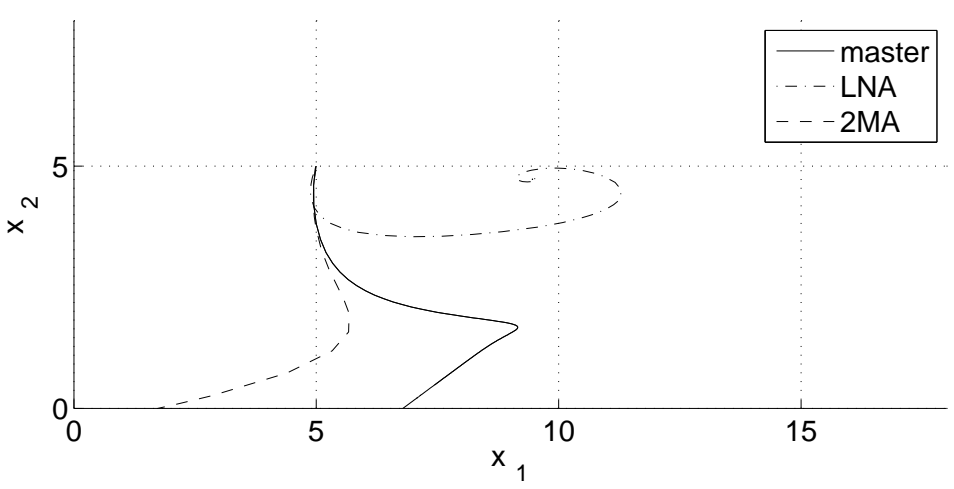

Fig. 10: Trajectories the expected values of the stable problem.

\subsubsection{The unstable system}

Let the parameters be $a=0.8, b=0.08, d=0.1$. Then the fixed point is unstable [32, p. 91] and the macroscopic solution has a limit cycle. The difference between the ME, LNA, and 2MA solutions with $\Omega_{1}=20$ is illustrated in Figure 11. The macroscopic solution and LNA solution approach the periodic orbit while the other two solutions end at the absorbing state.

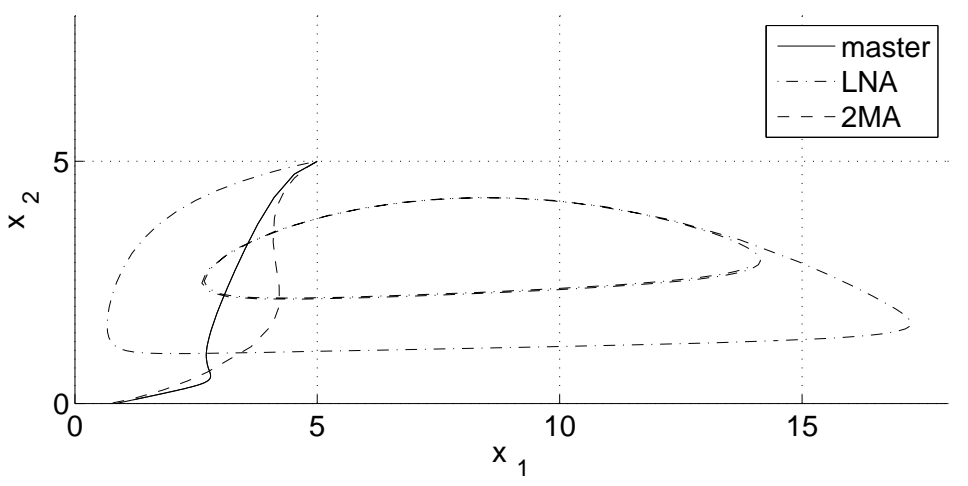

Fig. 11: Trajectories of the expected values of the unstable system at $\Omega_{1}=20, \Omega_{2}=10$.

Also for $\Omega_{1}=200$, the $2 \mathrm{MA}$ solution is closer to the ME solution initially in Figure 12.b. The limit cycle for the LNA solution is the same as in Figure 11 but with $x_{1}:=10 x_{1}, x_{2}:=10 x_{2}$. The ME is computed on a grid shown in Figure 12.a. The solution is sensitive to the grid resolution at $x_{2}=0$. When the grid size $\Delta x_{2}=1$ at the $x_{1}$-axis, then the absorbing state there is found. When $\Delta x_{2}=2$ there is no absorbing state at $x_{2}=0$ and a steady state for the expected value is reached at about $(115,25)$. The macroscopic and LNA solutions never reach the absorbing states unless $x_{2}(0)=0$. 


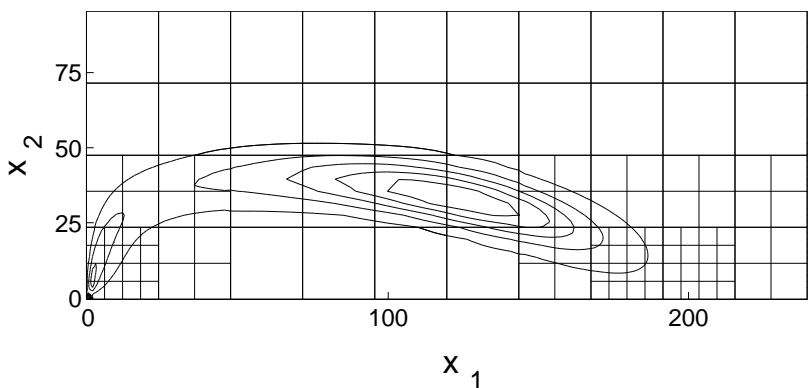

(a)

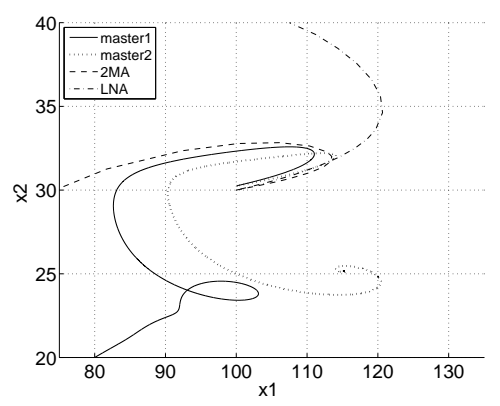

(b)

Fig. 12: The unstable problem. (a) A ME solution at $t=6.25$. (b) Trajectories of the expected values for the ME with $\Delta x_{2}=1$ (master1) and $\Delta x_{2}=2$ (master2).

While the 2MA captures the behavior of the ME in the beginning of the integration in Figure 12, it becomes unstable eventually with rapidly growing covariances and rapidly decreasing mean value. The divergence of the $2 \mathrm{MA}$ is confirmed by the estimates of the residual $\hat{\rho}^{k}$ in (36) in Figure 13. In the first phase, Figure 13.a, the 2MA is better than the LNA but later, Figure 13.b, the instability is clearly visible in the residual estimates for $2 \mathrm{MA}$.

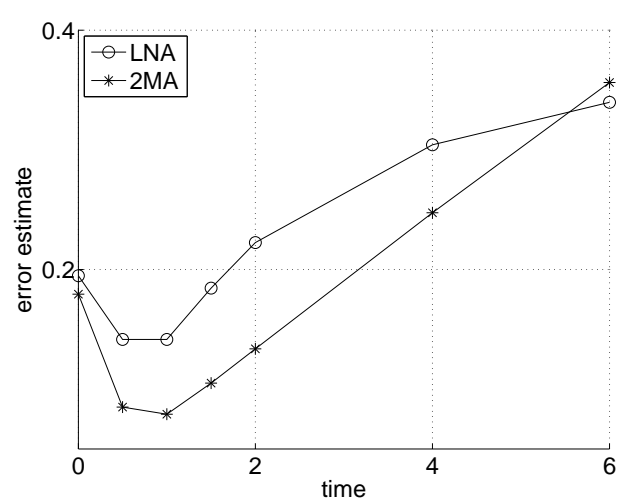

(a)

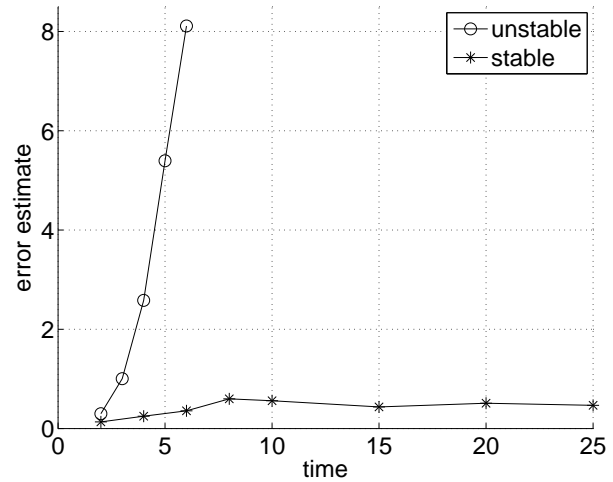

(b)

Fig. 13: Error estimates: (a) Comparison of LNA and 2MA in the initial phase of the stable problem. (b) Estimates for the stable and unstable problems and 2MA.

\subsection{Metabolites and enzymes}

The creation of two metabolites $A$ and $B$ is controlled by two enzymes $E_{A}$ and $E_{B}$ in a model in [8]. The reactions in the system are

$$
\begin{array}{lllll}
\emptyset \stackrel{w_{1}}{\longrightarrow} A & \emptyset \stackrel{w_{2}}{\longrightarrow} B & A+B \stackrel{w_{3}}{\longrightarrow} \emptyset & A \stackrel{w_{4}}{\longrightarrow} \emptyset & B \stackrel{w_{5}}{\longrightarrow} \emptyset \\
\emptyset \stackrel{w_{6}}{\longrightarrow} E_{A} & \emptyset \stackrel{w_{7}}{\longrightarrow} E_{B} & E_{A} \stackrel{w_{8}}{\longrightarrow} \emptyset & E_{B} \stackrel{w_{9}}{\longrightarrow} \emptyset &
\end{array}
$$


The propensities in the master equation for the nine reactions and the species $\mathbf{x}^{T}=\left(a, b, e_{A}, e_{B}\right)$ are

$$
\begin{aligned}
& \mathbf{n}_{1}^{T}=(-1,0,0,0), \quad w_{1}=\Omega \frac{k_{A} e_{A}}{\Omega+\frac{a}{K_{I}}}, \quad \mathbf{n}_{2}^{T}=(0,-1,0,0), \quad w_{2}=\Omega \frac{k_{B} e_{B}}{\Omega+\frac{b}{K_{I}}}, \\
& \mathbf{n}_{3}^{T}=(1,1,0,0), \quad w_{3}=k_{2} \Omega^{-1} a b, \quad \mathbf{n}_{4}^{T}=(1,0,0,0), \quad w_{4}=\mu a, \\
& \mathbf{n}_{5}^{T}=(0,1,0,0), \quad w_{5}=\mu b, \\
& \mathbf{n}_{7}^{T}=(0,0,0,-1), \quad w_{7}=\Omega \frac{k_{E_{B}}}{\Omega+\frac{b}{K_{R}}}, \quad \mathbf{n}_{8}^{T}=(0,0,1,0), \quad w_{8}=\mu e_{A}, \\
& \mathbf{n}_{9}^{T}=(0,0,0,1), \quad w_{9}=\mu e_{B} .
\end{aligned}
$$

The macroscopic propensities $v_{r}$ are obtained by letting $\Omega=1$ in (40). The coefficients are $k_{A}=k_{B}=0.3, k_{2}=0.001, K_{I}=60, K_{R}=30, k_{E_{A}}=$ $k_{E_{B}}=0.02, \mu=0.002$, and $\Omega=1$ in the experiments. The ME is solved with SSA taking the average of $10^{5}$ trajectories. The macroscopic equations (or reaction rate equations (RRE) in a biochemical problem) have a stable fixed point close to $(30.44,30.44,4.96,4.96)$, while the $2 \mathrm{MA}$ equations settles down at $(33.66,33.66,5.04,5.04)$. The time evolution of the expected values of the metabolite $A$ and the enzyme $E_{A}$ is found in Figure 14. Clearly, the 2MA solution is more accurate than the solution obtained with the RRE (and LNA) equations.

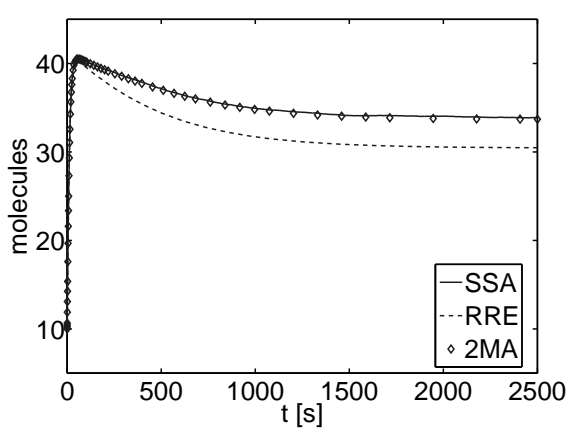

(a)

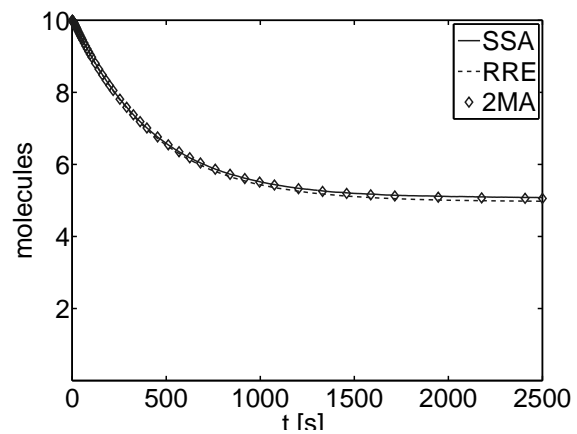

(b)

Fig. 14: Expected values computed with SSA, the RRE and 2MA. (a) The metabolite $A$. (b) The corresponding enzyme $E_{A}$.

The error estimate in (34) is approximated by a QMC method as in (37). Figure 15.a shows this estimate using ten randomized QMC sequences of $L=$ $2^{16} \approx 65 \cdot 10^{3}$ points. The number of points in $\mathcal{D}$ in Section 4 is about $100 \times$ $100 \times 30 \times 30=9 \cdot 10^{6}$. The residual error quickly settles at the steady state level. An estimate of the error in the QMC method using the randomized sequences [34] is compared in Figure 15.b for two different $L$. The quantity displayed is 
the width of a $95 \%$ confidence interval for $\hat{\rho}^{k}$. The error of the QMC summation is indeed small compared to the magnitude of the error estimate of the solution and a smaller $L$ would suffice.

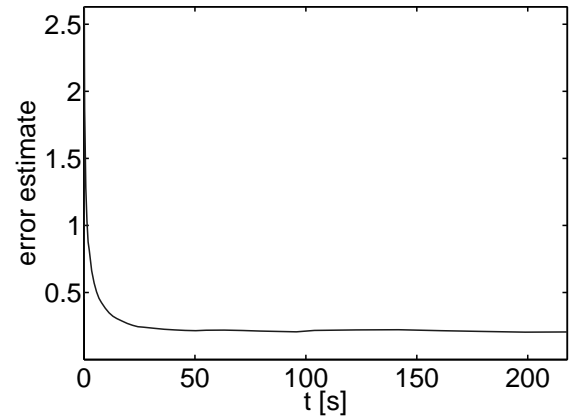

(a)

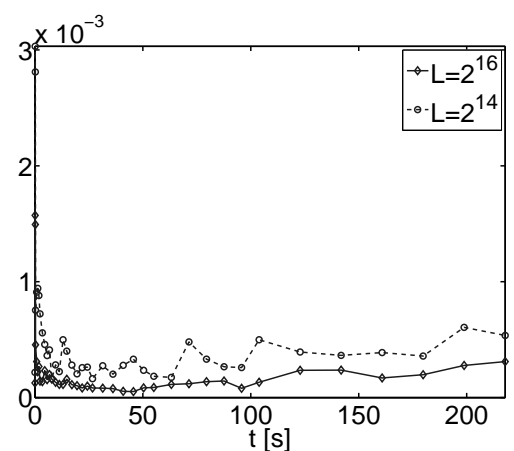

(b)

Fig. 15: (a) The residual error estimate of the 2MA solution. (b) The error of the QMC summation for two different numbers of points $L$.

In Table 1 we compare the correlation coefficients of the different species at $t=2500 \mathrm{~s}$ estimated from $10^{5}$ trajectories of SSA and computed with the 2MA equations. The 2MA solution agrees well with that of SSA.

\begin{tabular}{c|c|c|c|c}
\hline & $A$ & $B$ & $E_{A}$ & $E_{B}$ \\
\hline$A$ & 1 & -0.75 & 0.59 & -0.34 \\
\hline$B$ & -0.75 & 1 & -0.34 & 0.59 \\
\hline$E_{A}$ & 0.59 & -0.34 & 1 & 0.22 \\
\hline$E_{B}$ & -0.34 & 0.59 & 0.22 & 1 \\
\hline
\end{tabular}

\begin{tabular}{c|c|c|c|c}
\hline & $A$ & $B$ & $E_{A}$ & $E_{B}$ \\
\hline$A$ & 1 & -0.85 & 0.65 & -0.34 \\
\hline$B$ & -0.85 & 1 & -0.34 & 0.65 \\
\hline$E_{A}$ & 0.65 & -0.34 & 1 & 0.22 \\
\hline$E_{B}$ & -0.34 & 0.65 & 0.22 & 1 \\
\hline
\end{tabular}

Table 1: The correlation coefficients at $t=2500 \mathrm{~s}$ computed with SSA (left) and the 2MA equations (right).

\subsection{Circadian clock}

In a periodically changing environment, living cells have internal clocks to help them adapt to this environment. There are daily cycles of the light and annual cycles in the climate. Many organisms from small to large have developed molecular mechanisms for regulation of their biochemistry that is suitable for the circadian rhythm or $24 \mathrm{~h}$ cycle. A model for such a clock is found in [1, 40].

Consider the following 18 reactions for the nine molecular species 
A, $C, R, D_{a}, D_{a}^{\prime}, D_{r}, D_{r}^{\prime}, M_{a}, M_{a}^{\prime}$ modeling the circadian rhythm in [1]:

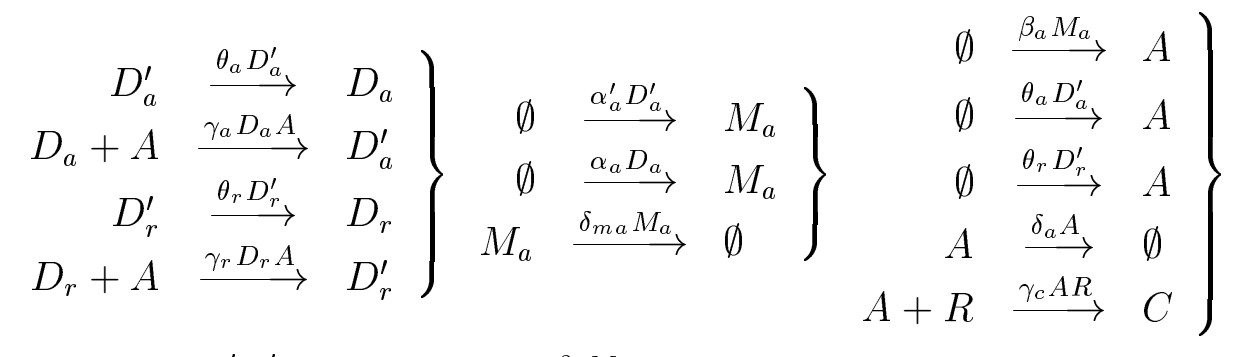

$$
\begin{aligned}
& \left.\left.\begin{array}{rll}
\emptyset & \stackrel{\alpha_{r}^{\prime} D_{r}^{\prime}}{\longrightarrow} & M_{r} \\
\emptyset & \stackrel{\alpha_{r} D_{r}}{\longrightarrow} & M_{r} \\
M_{r} & \stackrel{\delta_{m r} M_{r}}{\longrightarrow} & \emptyset
\end{array}\right\} \begin{array}{ccc}
\emptyset & \stackrel{\beta_{r} M_{r}}{\longrightarrow} & R \\
R & \stackrel{\delta_{r} R}{\longrightarrow} & \emptyset \\
C & \stackrel{\delta_{a} C}{\longrightarrow} & R
\end{array}\right\}
\end{aligned}
$$

All propensities $v_{r}$ are linear in the species except for three reactions with a quadratic dependence in (41). The corresponding $w_{r}$-form is the same as above for a linear $v_{r}$ and divided by $\Omega$ for a quadratic $v_{r}$.

The reaction constants are found in Table 2. The parameter $\delta_{r}$ will have values between 0 and 0.2 in the numerical experiments. The initial conditions are zero for all variables except for $D_{a}(0)=D_{r}(0)=1$.

\begin{tabular}{|c|c|c|c|c|c|c|c|c|c|}
\hline$\alpha_{a}$ & 50 & $\beta_{a}$ & 50 & $\gamma_{a}$ & 1 & $\delta_{m a}$ & 10 & $\theta_{a}$ & 50 \\
\hline$\alpha_{a}^{\prime}$ & 500 & $\beta_{r}$ & 5 & $\gamma_{r}$ & 1 & $\delta_{m r}$ & 0.5 & $\theta_{r}$ & 100 \\
\hline$\alpha_{r}$ & 0.01 & & & $\gamma_{c}$ & 2 & $\delta_{a}$ & 1 & & \\
\hline$\alpha_{r}^{\prime}$ & 50 & & & & & $\delta_{r}$ & - & & \\
\hline
\end{tabular}

Table 2: Parameters of the circadian clock.

The system is oscillatory but the period length depends on $\delta_{R}$ and $\Omega$. A subcritical Hopf bifurcation [38, p. 252] occurs for the macroscopic equations at $\delta_{R c} \approx 0.096$ and the fixed point $(C, R) \approx(325,90)$. Two complex conjugate eigenvalues of the Jacobian of the system leave the left half of the complex plane and enter the right half plane. When $\delta_{R}<\delta_{R c}$, there is a stable fixed point inside an unstable limit cycle but for $\delta_{R}>\delta_{R c}$ the limit cycle disappears and the fixed point is unstable. The unstable limit cycle encompasses a larger and larger area the smaller $\delta_{R}$ is. In Figure 16.a, the outer limit cycle and a part of a trajectory starting close to the unstable fixed point are displayed. One trajectory outside the unstable limit cycle approaches the outer limit cycle and the other trajectory inside the unstable limit cycle converges to the fixed point in Figure 16.b. The outer limit cycle disappears when $\delta_{R} \approx 0.85$. 


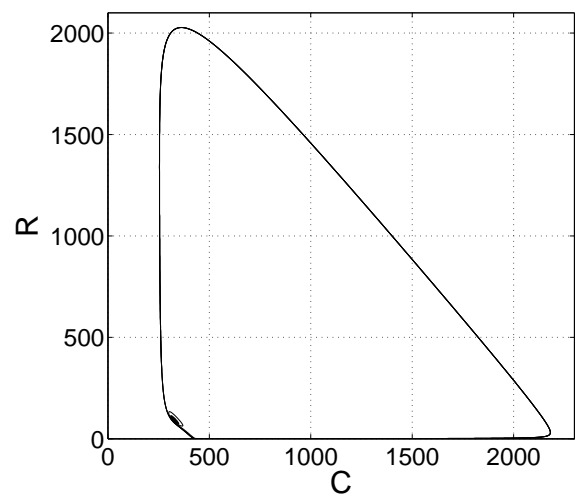

(a)

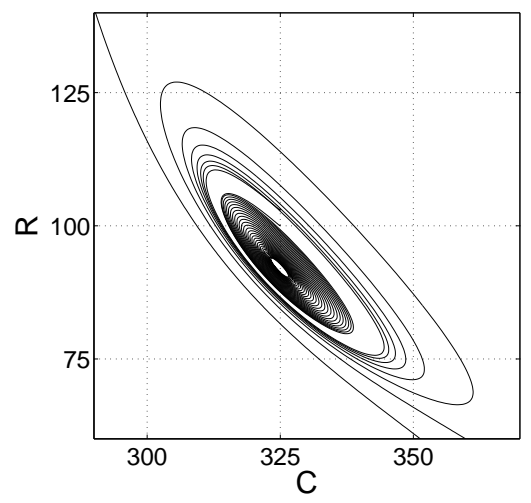

(b)

Fig. 16: (a) The outer limit cycle and the unstable fixed point at $(C, R) \approx(325,90)$ for $\delta_{R}=0.097$. (b) A close-up view of the stable fixed point and the unstable limit cycle for $\delta_{R}=0.095$.

The system is solved with the LNA and the 2MA. The period is determined by the macroscopic equations for the mean values with LNA (5). With 2MA, there is a feedback from the covariances to the mean value equation (19), which is not present in (5), introducing a dependence of the period on $\Omega$. The larger $\Omega$ is, the closer the 2MA solution is to the LNA solution according to the analysis in Section 3. This is confirmed in Figure 17. An example of an oscillatory solution computed with $2 \mathrm{MA}$ is found to the left in the figure. Then the period length is displayed for varying $\delta_{R}$ and $\Omega$. One trajectory of the system is simulated with SSA and an average period is recorded for ten peaks for different $\delta_{R}$ and $\Omega$ in Figure 18. The agreement between the 2MA and the SSA simulations is good.

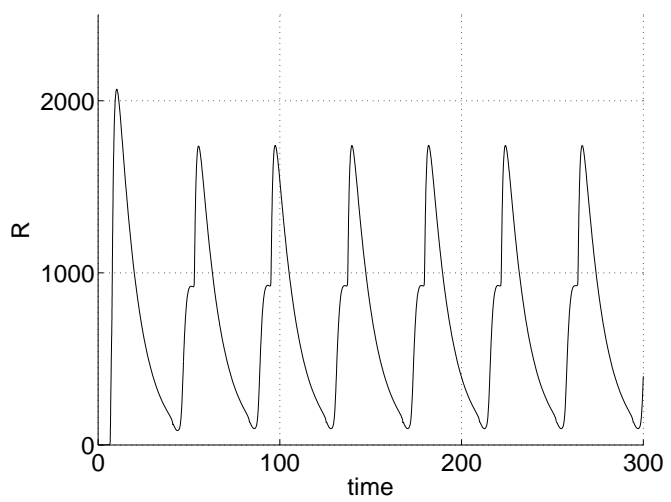

(a)

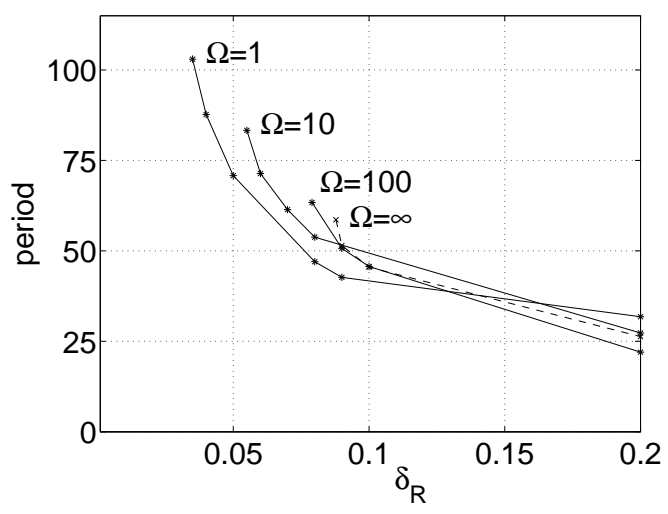

(b)

Fig. 17: (a) The period length with $2 \mathrm{MA}$ is about 43 when $\delta_{R}=0.09$ and $\Omega=1$. (b) The period length with LNA (dashed, $\Omega=\infty$ ), and 2MA with different $\Omega$ (solid). 


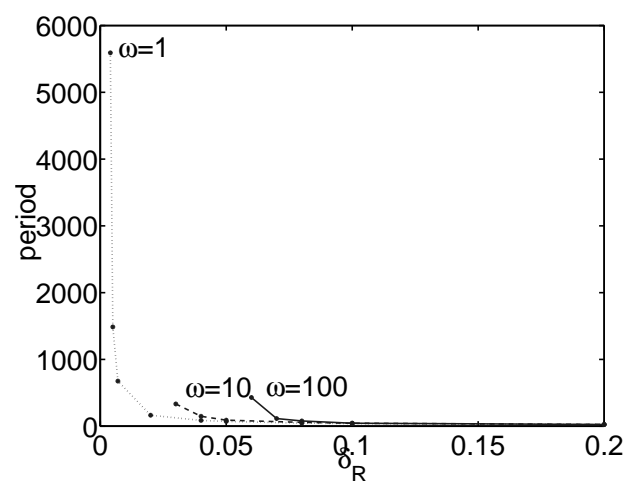

(a)

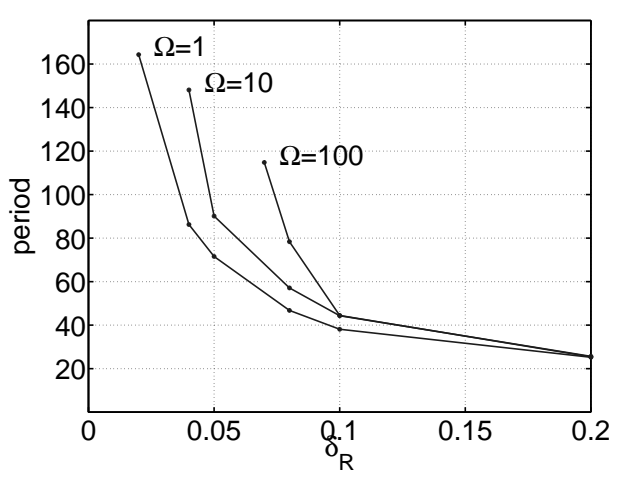

(b)

Fig. 18: (a) The average period lengths obtained with SSA. (b) A close-up view of the period lengths.

\section{Conclusions}

We have considered a hierarchy of models from coarse to fine: the macroscopic model, the linear noise approximation (LNA), the two-moment approximation (2MA), and the master equation (ME). They have been compared theoretically and in numerical experiments. Both approximations require the solution of two systems of ODEs for the expected values and the covariances. The computational work grows as a polynomial of low order with the number of species in the system. The equation for the expected value of the LNA is the macroscopic equation.

A system size parameter $\Omega$ is introduced. When $\Omega$ increases, the solution of the 2MA converges to the solution of the LNA. Furthermore, the expected value of the PDF of the ME converges to the macroscopic solution when $\Omega \rightarrow \infty$. The residual error of the approximations is measured by evaluating a discretization of the ME. The LNA and 2MA are compared to the ME solutions in four different examples. In some cases, the LNA and the 2MA generate accurate solutions (Figures 1,4) but in other cases the deviations from the ME solutions are large (Figure 3). The 2MA is accurate when the moments higher than two are small. Also, the 2MA solutions follow the expected value of the ME solutions better than the LNA solutions due to the influence of the variance and $\Omega$ in the equations for the expected values (Figures 7, 9, 10, 11, 12, 14, 17, 18). This behavior is very well captured by the residual calculations (Figures 4, 13, 15). In most cases, the 2MA has at least the right qualitative behavior compared to the ME solutions but the LNA often fails.

\section{References}

[1] N. Barkai, S. Leibler, Circadian clocks limited by noise, Nature, 403 
(2000), p. 267-268.

[2] R. E. Caflisch, Monte Carlo and quasi-Monte Carlo methods, Acta Numerica, 1998, p. 1-49.

[3] Y. Cao, D. Gillespie, L. Petzold, Multiscale stochastic simulation algorithm with stochastic partial equilibrium assumption for chemically reacting systems, J. Comput. Phys., 206 (2005), p. 395-411.

[4] W.-Y. Chen, A. Bokka, Stochastic modeling of nonlinear epidemiology, J. Theor. Biol., 234 (2005), p. 455-470.

[5] U. Dieckmann, P. Marrow, R. Law, Evolutionary cycling in predatorprey interactions: Population dynamics and the Red Queen, J. Theor. Biol., 176 (1995), p. 91-102.

[6] J. Dieudonné, Foundations of Modern Analysis, Academic Press, New York, 1969.

[7] W. E, D. Liu, E. Vanden-Eijnden, Nested stochastic simulation algorithm for chemical kinetic systems with multiple time scales, J. Comput. Phys., 221 (2007), p. 158-180.

[8] J. Elf, P. Lötstedt, P. SJöberg, Problems of high dimension in molecular biology, in High-dimensional problems - Numerical treatment and applications, ed. W. Hackbusch, Proceedings of the 19th GAMM-Seminar Leipzig 2003, p. 21-30, available at http://www.mis.mpg.de/conferences/gamm/2003/.

[9] J. Elf, M. Ehrenberg, Fast evaluation of fluctuations in biochemical networks with the linear noise approximation, Genome Res., 13 (2003), p. $2475-2484$.

[10] J. Elf, J. Paulsson, O. G. Berg, M. Ehrenberg, Near-critical phenomena in intracellular metabolite pools, Biophys. J., 84 (2003), p. 154-170.

[11] C. Escudera, J. Buceta, F. J. de la Rubia, K. Lindenberg, Extinction in population dynamics, Phys. Rev. E, 69 (2004), 021908.

[12] S. EngBlom, Computing the moments of high dimensional solutions of the master equation, Appl. Math. Comput., 180 (2006), p. 498-515.

[13] B. Engquist, O. Runborg, Computational high frequency wave propagation, Acta Numerica, (2003), p. 181-266.

[14] S. N. Ethier, T. G. Kurtz, Markov Processes, Characterization and Convergence, John Wiley, New York, 1986. 
[15] H. FAure, Discrépance de suites associées à un système de numération (en dimension s), Acta Aritm., 41 (1982), p. 337-351.

[16] L. Ferm, P. Lötstedt, P. SjöBerg, Conservative solution of the FokkerPlanck equation for stochastic chemical reactions, BIT, 46 (2006), p. S61S83.

[17] R. F. Fox, J. Keizer, Amplification of intrinsic fluctuations by chaotic dynamics in physical systems, Phys. Rev. A, 43 (1991), p. 1709-1720.

[18] C. W. Gardiner, Handbook of Stochastic Methods, 3rd ed., Springer, Berlin, 2004.

[19] M. B. Giles And E. Süli, Adjoint methods for PDEs: a posteriori error analysis and postprocessing by duality, Acta Numerica, 11 (2002), p. 145236.

[20] D. T. Gillespie, A general method for numerically simulating the stochastic time evolution of coupled chemical reactions, J. Comput. Phys., 22 (1976), p. 403-434.

[21] D. Givon, R. Kupferman, A. Stewart, Extracting macroscopic dynamics: model problems and algorithms, Nonlinearity, 17 (2004), p. R55-R127.

[22] E. Hairer, S. P. Nørsett, G. Wanner, Solving Ordinary Differential Equations I, Nonstiff Problems, 2nd ed., Springer-Verlag, Berlin, 1993.

[23] H. S. Hong, F. J. Hickernell, Algorithm 823: Implementing scrambled digital sequences, ACM Trans. Math. Softw., 29 (2003), p. 95-109.

[24] N. G. van Kampen, The expansion of the master equation, Adv. Chem. Phys., 34 (1976), p. 245-309.

[25] N. G. van Kampen, Stochastic Processes in Physics and Chemistry, NorthHolland, Amsterdam, 1992.

[26] T. G. Kurtz, Solutions of ordinary differential equations as limits of pure jump Markov processes, J. Appl. Probab., 7 (1970), p. 49-58.

[27] T. G. Kurtz, Limit theorems for sequences of jump Markov processes approximating ordinary differential processes, J. Appl. Probab., 7 (1971), p. $344-356$.

[28] P. LÖtstedt, L. Ferm, Dimensional reduction of the Fokker-Planck equation for stochastic chemical reactions, Multiscale Meth. Simul., 5 (2006), p. 593-614. 
[29] H. H. McAdams, A. Arkin, Stochastic mechanisms in gene expression, Proc. Natl. Acad. Sci. USA, 94 (1997), p. 814-819.

[30] H. H. McAdams, A. Arkin, It's a noisy business. Genetic regulation at the nanomolar scale, Trends Gen., 15 (1999), p. 65-69.

[31] A. J. McKane, T. J. Newman, Stochastic models in population biology and their deterministic analogs, Phys. Rev. E, 70 (2004), 041902.

[32] J. D. Murray, Mathematical Biology. I: An Introduction, 3rd ed., Springer, New York, 2002.

[33] J. T. Oden, S. Prudhomme, Estimation of modeling error in computational mechanics, J. Comput. Phys., 182 (2002), p. 496-515.

[34] A. B. Owen, Monte Carlo variance of scrambled net quadrature, SIAM J. Numer. Anal., 34 (1997), p. 1884-1910.

[35] H. Risken, The Fokker-Planck Equation, 2nd ed., Springer, Berlin, 1996.

[36] P. Sjöвerg, P. LÖtstedt, J. ElF, Fokker-Planck approximation of the master equation in molecular biology, Technical report 2005-044, Dept of Information Technology, Uppsala University, Uppsala, Sweden, 2005, to appear in Comput. Vis. Sci., available at http://www.it.uu.se/research/reports/2005-044/.

[37] N. Stollenwerk, V. A. A. Jensen, Meningitis, pathogenicity near criticality: the epidemiology of meningococcal disease as a model for accidental pathogens, J. Theor. Biol., 222 (2003), p. 347-359.

[38] S. H. Strogatz, Nonlinear Dynamics and Chaos, Perseus Books, Cambridge, MA, 1994.

[39] S. SuccI, The Lattice Boltzmann Equation for Fluid Dynamics and Beyond, Clarendon Press, Oxford, 2001.

[40] J. M. G. Vilar, H. Y. Kueh, N. Barkai, S. Leibler, Mechanisms of noise-resistance in genetic oscillators, Proc. Nat. Acad. Sci. USA, 99 (2002), p. $5988-5992$.

[41] W. Weidlich, Sociodynamics. A Systematic Approach to Mathematical Modelling in the Social Sciences, Taylor and Francis, London, 2000.

[42] W. WeIDLICH, Thirty years of sociodynamics. An integrated strategy of modelling in the social sciences: applications to migration and urban evolution, Chaos, Solit. Fract., 24 (2005), p. 45-56.

[43] D. C. Wilcox, Turbulence Modeling for CFD, DCW Industries, Inc., La Cañada, CA, 1994. 